\title{
On a conjecture concerning the Petersen graph
}

\author{
Donald Nelson \\ Department of Mathematical Sciences \\ Middle Tennessee State University \\ Murfreesboro, TN 37132, USA \\ dnelson@mtsu.edu \\ Neil Robertson* \\ Department of Mathematics \\ Ohio State University \\ Columbus, OH 43210, USA \\ robertso@math.ohio-state.edu
}

\author{
Michael D. Plummer \\ Department of Mathematics \\ Vanderbilt University \\ Nashville, TN 37240, USA \\ michael.d.plummer@vanderbilt.edu \\ Xiaoya $\mathrm{Zha}^{\dagger}$ \\ Department of Mathematical Sciences \\ Middle Tennessee State University \\ Murfreesboro, TN 37132, USA \\ xzha@mtsu.edu
}

Submitted: Oct 4, 2010; Accepted: Jan 10, 2011; Published: Jan 19, 2011

Mathematics Subject Classifications: 05C38, 05C40, 05C75

\begin{abstract}
Robertson has conjectured that the only 3-connected, internally 4-connected graph of girth 5 in which every odd cycle of length greater than 5 has a chord is the Petersen graph. We prove this conjecture in the special case where the graphs involved are also cubic. Moreover, this proof does not require the internal-4-connectivity assumption. An example is then presented to show that the assumption of internal 4-connectivity cannot be dropped as an hypothesis in the original conjecture.

We then summarize our results aimed toward the solution of the conjecture in its original form. In particular, let $G$ be any 3-connected internally-4connected graph of girth 5 in which every odd cycle of length greater than 5 has a chord. If $C$ is any girth cycle in $G$ then $N(C) \backslash V(C)$ cannot be edgeless, and if $N(C) \backslash V(C)$ contains a path of length at least 2, then the conjecture is true. Consequently, if the conjecture is false and $H$ is a counterexample, then for any girth cycle $C$ in $H, N(C) \backslash V(C)$ induces a nontrivial matching $M$ together with an independent set of vertices. Moreover, $M$ can be partitioned into (at most) two disjoint non-empty sets where we can precisely describe how these sets are attached to cycle $C$.
\end{abstract}

\footnotetext{
* work supported by NSF Grant DMS-0354554

$\dagger$ work supported by NSA Grant H98230-09-01-0041
} 


\section{Introduction and Terminology.}

This paper is motivated by the following conjecture due to Robertson:

Conjecture 1.1: The only 3-connected, internally 4-connected, girth 5 graph in which every odd cycle of length greater than 5 has a chord is the Petersen graph.

Since its discovery at the end of the nineteenth century, the Petersen graph has been cited as an example, and even more often as a counterexample, in nearly every branch of graph theory. These occurrences could fill a book and in fact have; see [HoSh]. We will not attempt to give a complete list of the appearances of this remarkable graph in print, but let us mention a few of the more recent applications. Henceforth, we shall denote the Petersen graph by $P_{10}$.

Let us now adopt the following additional notation. If $u$ and $v$ are distinct vertices in $P_{10}$, the graph formed by removing vertex $v$ will be denoted $P_{10} \backslash v$ and, if $u$ and $v$ are adjacent, the subgraph obtained by removing edge $u v$ will be denoted by $P_{10} \backslash u v$. Other notation and terminology will be introduced as needed.

It is a well-known fact that every Cayley graph is vertex-transitive, but the converse is false, the smallest counterexample being $P_{10}$. (Cf. [A].) In their studies of vertextransitive graphs [LS, Sc], the authors present four interesting classes of non-Cayley graphs and digraphs (generalized Petersen, Kneser, metacirculant and quasi-Cayley) and all four classes contain $P_{10}$.

The Petersen graph has long played an important role in various graph traversability problems. It is known to be the smallest hypohamiltonian graph [GHR]. It is also one of precisely five known connected vertex-transitive graphs which fail to have a Hamilton cycle. It does, however, possess a Hamilton path. Lovász [L1] asked if every connected vertex-transitive graph contains a Hamilton path. This question has attracted considerable attention, but remains unsolved to date. (Cf. [KM1, KM2].)

One of the earliest alternative statements of the 4-color conjecture was due to Tait [Ta]: Every cubic planar graph with no cut-edge is 3-edge-colorable. The Petersen graph $P_{10}$ is the smallest nonplanar cubic graph that is not 3 -edge colorable. Some eleven years before the 4-color problem was settled [AH1, AH2], Tutte [Tu1, Tu2] formulated the following stronger conjecture about cubic graphs:

Conjecture 1.2: Every cubic cut-edge free graph containing no $P_{10}$-minor is 3 -edgecolorable.

A cubic graph with no cut-edge which is not 3-edge-colorable is called a snark. Not surprisingly, in view of the preceding conjecture of Tutte, much effort has been devoted to the study of snarks and many snark families have been discovered. (Cf. [Wa, WW, CMRS].) However, to date, all contain a Petersen minor. A proof has been announced by Robertson, Sanders, Seymour and Thomas [Th, TT], but has not yet appeared.

Note that there is a relationship between the question of Lovász and 3-edgecolorings in that for cubic graphs, the existence of a Hamilton cycle guarantees an edge coloring in three colors. Actually, there are only two known examples of connected 
cubic vertex-transitive graphs which are not 3-edge-colorable of which $P_{10}$ is one and the other is the cubic graph derived from $P_{10}$ by replacing each vertex by a triangle. (Cf. $[\mathrm{Po}]$.) (The latter graph is known as the inflation or the truncation of $P_{10}$.)

Note also that a 3-edge-coloring of graph $G$ is equivalent to being able to express the all-1's vector of length $|E(G)|$ as the sum of the incidence vectors of three perfect matchings. Seymour [Se1] was able to prove a relaxation of Tutte's conjecture by showing that every cubic bridgeless graph with no $P_{10}$-minor has the property that the edge-incidence vector of all-1's can be expressed as an integral combination of the perfect matchings of $G$. Lovász [L2] later derived a complete characterization, in which the Petersen graph plays a crucial role, of the lattice of perfect matchings of any graph.

In connection with covering the edges of a graph by perfect matchings, we should also mention the important - and unsolved - conjecture of Berge and Fulkerson [F; see also Se1, Zhan].

Conjecture 1.3: Every cubic cut-edge free graph $G$ contains six perfect matchings such that each edge of $G$ is contained in exactly two of the matchings.

The Petersen graph, in fact, has exactly six perfect matchings with this property.

Drawing on his studies of face-colorings, Tutte also formulated a related conjecture for general (i.e., not necessarily cubic) graphs in terms of integer flows.

Conjecture 1.4: Every cut-edge free graph containing no subdivision of $P_{10}$ admits a nowhere-zero 4-flow.

This conjecture too has generated much interest. For cubic graphs, Conjecture 1.2 and Conjecture 1.4 are equivalent since in this case a 3-edge-coloring is equivalent to a 4-flow . The 5-flow analogue for cubic graphs, however, has been proved by Kochol [Ko].

Theorem 1.5: If $G$ is a cubic cut-edge free graph with no Petersen minor, $G$ has a nowhere-zero 5-flow.

Another partial result toward the original conjecture is due to Thomas and Thomson $[\mathrm{TT}]$ :

Theorem 1.6: Every cut-edge free graph without a $P_{10} \backslash e$-minor has a nowhere-zero 4-flow.

This result generalizes a previous result of Kilakos and Shepherd [KS] who had derived the same conclusion with the additional hypothesis that the graphs be cubic.

The original (not necessarily cubic) 4-flow conjecture remains unsolved.

Yet another widely studied problem is the cycle double conjecture. A set of cycles in a graph $G$ is a cycle double cover if every edge of $G$ appears in exactly two of the cycles in the set. The following was conjectured by Szekeres [Sz] and, independently, by Seymour [Se2]. It remains open.

Conjecture 1.7: Every connected cut-edge free graph contains a cycle double cover. 
The following variation involving $P_{10}$ was proved by Alspach, Goddyn and Zhang [AGZ].

Theorem 1.8: Every connected cut-edge free graph with no $P_{10}$-minor has a cycle double cover.

For much more on the interrelationships of edge-colorings, flows and cycle covers, the interested reader is referred to [Zhan, Ja].

An embedding of a graph $G$ in 3-space is said to be flat if every cycle of the graph bounds a disk disjoint from the rest of the graph. Sachs [Sa] conjectured that a graph $G$ has a flat embedding in 3-space if and only if it contains as a minor none of seven specific graphs related to $P_{10}$. His conjecture was proved by Robertson, Seymour and Thomas [RST3].

Theorem 1.9: A graph $G$ has a flat embedding if and only if it has no minor isomorphic to one of the seven graphs of the 'Petersen family' obtained from $P_{10}$ by $\mathrm{Y}-\Delta$ and $\Delta-\mathrm{Y}$ transformations. (the complete graph $K_{6}$ is one of these seven graphs.)

A smallest graph with girth $g$ and regular of degree $d$ is called a $(d, g)$-cage. The unique $(3,5)$-cage is $P_{10}$. This observation was proved by Tutte [Tu3] under a more stringent definition of "cage".

Any smallest graph which is regular of degree $d$ and has diameter $k$ (if it exists) is called a Moore graph of type $(d, k)$. For $k=2$, Moore graphs exist only for $d=2,3,7$ and possibly 57 . The unique Moore graph of type $(3,2)$ is $P_{10}$. (Cf. [HoSi].)

A graph $G$ is said to be distance-transitive if for every two pairs of vertices $\{v, w\}$ and $\{x, y\}$ such that $d(u, v)=d(x, y)$ (where $d$ denotes distance), there is an automorphism $\sigma$ of $G$ such that $\sigma(v)=x$ and $\sigma(w)=y$. There are only twelve finite cubic distance-transitive graphs and $P_{10}$ is the only one with diameter 2 and girth 5 . (Cf. [BS].)

Distance-transitive graphs form a proper subclass of another important graph class called distance-regular graphs. (Cf. [BCN].) These graphs are closely related to the association schemes of algebraic combinatorics.

A closed 2-cell surface embedding of a graph $G$ is called strong (or circular). The following conjecture is folklore which appeared in literature as early as in 1970s (Cf. [H, $\mathrm{LR}])$.

Conjecture 1.10: Every 2-connected graph has a strong embedding in some surface.

(Note that, for cubic graphs, this conjecture is equivalent to the cycle-double-cover conjecture.) (Cf. [Zhan, Corollary 7.1.2].)

Ivanov and Shpectorov [I, IS] have investigated certain so-called Petersen geometries associated with the sporadic simple groups. The smallest of these geometries is associated with $P_{10}$. 
The following conjecture of Dirac was proved by Mader.

Theorem 1.11 [M1]: Every graph $G$ with at least $3|V(G)|-5$ edges (and at least 3 vertices) contains a subdivision of $K_{5}$.

One of the main tools used in proving this is another of Mader's own theorems.

Theorem 1.12 [M2]: If $G$ has girth at least 5 , at least 6 vertices and at least $2|V(G)|-5$ edges, then $G$ either contains a subdivision of $K_{5} \backslash e$ or $G \cong P_{10}$.

Our plan of attack is to proceed as follows. In Section 2, we present several lemmas of a technical nature. In Section 3, we prove the conjecture for cubic graphs without using the internal-4-connectivity assumption. We then close the section by presenting infinitely many examples of a graphs which are 3-connected of girth 5 and in which every odd cycle of length greater than 5 has a chord, but which are not the Petersen graph. These examples led us to invoke the additional assumption of internal-4-connectivity.

Let $H$ be a subgraph of a graph $G$. Denote by $N^{\prime}(H)$ the set of neighbors of vertices in $H$ which are not themselves in $H$. We also use $N^{\prime}(H)$ to denote the subgraph induced by $N^{\prime}(H)$ (this will not cause any confusion in this paper). Let $G$ be a 3-connected internally-4-connected graph $G$ having girth 5 in which every odd cycle of length greater than 5 has a chord. Let $C$ be a 5 -cycle in $G$. We then proceed to focus our attention on the structure of the subgraph induced by $N^{\prime}(C)$.

In Section 4, we show that $N^{\prime}(C)$ cannot be edgeless. In Section 5, we show that if $N^{\prime}(C)$ contains a path of length at least 3 , then $G \cong P_{10}$. In Section 6 we undertake the lengthier task of showing that if $N^{\prime}(C)$ contains a path of length 2 , then $G \cong P_{10}$. In summary then, we will reduce the conjecture to the case when $N^{\prime}(C)$ is the disjoint union of a nonempty matching and a possibly empty edgeless subgraph. Moreover, the matching must be attached to the 5 -cycle $C$ only in certain restricted ways. We will summarize these details in Section 7 . 


\section{Some technical lemmas.}

Suppose $H$ is a subgraph of a graph $G$ and $x \in V(G)$. Denote $N(x)=\{v \in V(G)$ : $v x \in E(G)\}, N(H)=\{v \in V(G): u v \in E(G)$ for some $u \in V(H)\}$ and $N^{\prime}(H)=$ $N(H) \backslash V(H)$. Define $N^{\prime}(H, x)=N(x) \backslash V(H)$. Note that in general $N(x)$ does not contain $x$ and so $N^{\prime}(x)=N(x)$ when $x$ is not in $V(H)$. If $V(H)=\left\{x_{1}, x_{2}, \ldots, x_{t}\right\}$, we will write $N_{i}^{\prime}$ for $N^{\prime}\left(x_{i}\right) \backslash V(H)=N\left(H, x_{i}\right)$, where $1 \leq i \leq t$, ignoring the dependency on $H$. Since all graphs $G$ in this paper are assumed to have girth $5, N(x)$ is an independent set, for all $x \in V(G)$, and hence any $N^{\prime}(x)$ in this paper will be independent as well.

Let $G$ be a graph and $H$ a proper subgraph of $G$. If $e=x y$ is an edge of $G$ not belonging to $H \cup V\left(N^{\prime}(H)\right)$, but joining two vertices $x$ and $y$ of $N^{\prime}(H)$, we call $e$ an edge-bridge of $H \cup V\left(N^{\prime}(H)\right)$. Let $D$ be a component of $G \backslash\left(V(H) \cup V\left(N^{\prime}(H)\right)\right)$. If there exists a vertex $w \in N^{\prime}(H)$ which is adjacent to some vertex of $D$, we will say that $w$ is a vertex of attachment for $D$ in $N^{\prime}(H)$. If $D$ is a component of $G \backslash\left(V(H) \cup V\left(N^{\prime}(H)\right)\right)$ and $B$ consists of $D$, together with all of its vertices of attachment in $H$, we call $B$ a non-edge-bridge of $H$. Furthermore, any vertex of bridge $B$ which is not a vertex of attachment will be called an interior vertex of $B$. Clearly, any path from an interior vertex of $B$ to a vertex in $H$ passes through a vertex of attachment of $B$.

We now further classify the non-edge-bridges of $H \cup N^{\prime}(H)$ as follows. If such a non-edge-bridge has all of its vertices of attachment in the same $N^{\prime}(x)$, we will call it a monobridge and if $x=x_{i}$ we will often denote it by $B_{i}$. Now suppose that $N^{\prime}\left(x_{i}\right) \cap$ $N^{\prime}\left(x_{j}\right)=\emptyset$, for all $x_{i} \neq x_{j} \in V(H)$. Then if $x_{i} \neq x_{j} \in V(H)$, a bibridge $B_{i, j}$ of $H \cup N^{\prime}(H)$ is a bridge which is not a monobridge, but has all of it vertices of attachment in the two sets $N_{i}^{\prime}$ and $N_{j}^{\prime}$.

Two distinct vertices $x$ and $y$ in a subgraph $H$ will be called a co-bridge pair in $H$ if there exists a non-edge bridge $B$ of $H \cup N^{\prime}(H)$ such that $B$ has an attachment in $N^{\prime}(x)$ and an attachment in $N^{\prime}(y)$. If two vertices of $H$ are not a co-bridge pair, they will be called a non-co-bridge pair in $H$.

Two distinct non-adjacent vertices $x$ and $y$ in a subgraph $H$ will be called wellconnected in $H$ if $x$ and $y$ are non-adjacent and there exist two induced paths in $H$ joining $x$ and $y$ one of which is of odd length at least 3 and the other of even length at least 2 .

Lemma 2.1: Let $G$ be a 3-connected graph of girth five in which every odd cycle of length greater than 5 contains a chord. Let $H$ be a subgraph of $G$ and $x, y$, two vertices of $H$ such that

(1) $x$ and $y$ are well-connected in $H$,

(2) $N^{\prime}(x) \cap N^{\prime}(y)=\emptyset$ and

(3) there exists no edge-bridge having one endvertex in $N^{\prime}(x)$ and the other in $N^{\prime}(y)$.

Then $x$ and $y$ are a non-co-bridge pair in $H$.

Proof: Suppose, to the contrary, that $B$ is a non-edge bridge of $H \cup N^{\prime}(H)$ with vertex $u$ a vertex of attachment of $B$ in $N^{\prime}(x)$ and $v$ a vertex of attachment of $B$ in $N^{\prime}(y)$. Let 
$P_{u v}$ be a shortest path in $B$ joining $u$ and $v$. Since $B$ is a non-edge bridge, $P_{u v}$ contains at least two edges. Let $Q_{x y}$ and $Q_{x y}^{\prime}$ be induced paths in $H$ joining $x$ and $y$ and having opposite parity. Then let $P=P_{u v} \cup Q_{x y} \cup\{u x, v y\}$ and $P^{\prime}=P_{u v} \cup Q_{x y}^{\prime} \cup\{u x, v y\}$. Then both $P$ and $P^{\prime}$ are chordless and one of them is an odd cycle of length at least 7 , a contradiction.

Lemma 2.2: Suppose $G$ is 3 -connected and has girth 5. Let $C$ be any cycle in $G$ of length 5 . Then the subgraph induced by $N^{\prime}(C)$ has maximum degree 2 .

Proof: This is an easy consequence of the girth 5 assumption.

Lemma 2.3: Suppose $G$ is 3 -connected, has girth 5 and all odd cycles of length greater than 5 have a chord. Then $G$ contains no cycle of length 7 .

Proof: Suppose $C$ is a 7 -cycle in $G$. Then $C$ must have a chord which then lies in a cycle of length at most 4 , a contradiction.

We will also need the next three results on traversability in $P_{10}, P_{10} \backslash v$ and $P_{10} \backslash u v$. At this point we remind the reader that the Petersen graph is both vertex- and edgetransitive. In the proof of the following two lemmas and henceforth we shall make use of these symmetry properties.

Lemma 2.4: Let $P_{10}$ denote the Petersen graph and let $x$ and $y$ be any two non-adjacent vertices in $P_{10}$. Then there exist

(i) a unique induced path of length 2 joining $x$ and $y$;

(ii) exactly two internally disjoint induced paths of length 3 joining $x$ and $y$; and

(iii) exactly two internally disjoint induced paths of length 4 joining $x$ and $y$.

(iv) Moreover if $z$ is adjacent to both $x$ and $y$, then these induced paths of length 3 and 4 do not pass through $z$.

Proof: This is easily checked.

Lemma 2.5: (i) Let $P_{10} \backslash v$ be the Petersen graph with one vertex $v$ removed. Then for every pair of non-adjacent vertices $x$ and $y$, there exist induced paths of length 3 and 4 joining them.

(ii) Let $P_{10} \backslash u v$ denote the Petersen graph with a single edge $u v$ removed. Then for every pair of non-adjacent vertices $x$ and $y$, there exists an induced path of length 4 and either an induced path of length 3 or one of length 5 .

(iii) Moreover, in both (i) and (ii) if $z$ is a vertex adjacent to both $x$ and $y$, these paths do not pass through $z$.

Proof: The existence of induced paths of length 3 and 4 is a direct consequence of Lemma 2.4 since in $P_{10}$ there are two internally disjoint paths of each type.

If $z$ is incident to both $x$ and $y$, then any induced path joining $x$ and $y$ and passing through $z$ has length exactly 2 . Therefore any induced path joining $x$ and $y$ of length 3 or 4 does not pass through $z$. 
Corollary 2.6: In any of the three graphs $P_{10}, P_{10} \backslash v$ and $P_{10} \backslash u v$, if $x_{i}$ and $x_{j}$ are any pair of distinct non-adjacent vertices, then they are well-connected.

\section{The cubic case.}

In this section we prove the conjecture for graphs which are 3-connected and cubic, have girth 5 and in which every odd cycle of length greater than 5 has a chord. Note that we do not assume internal-4-connectivity in this section.

We begin by treating the case in which for some girth cycle $C, N^{\prime}(C)$ contains a path of length at least 3 . Then by eliminating in sequence five cases corresponding to five possible subgraphs, we arrive at our final result. Although the approach in these five cases is much the same, nevertheless each of the final four makes use of its predecessor in the sequence.

Lemma 3.1: Suppose $G$ is a cubic 3-connected graph of girth 5 in which every odd cycle of length greater than 5 has a chord. Let $C$ be a 5 -cycle in $G$. Then if $N^{\prime}(C)$ contains a path of length at least $3, G \cong P_{10}$.

Proof: Let $C=x_{1} x_{2} x_{3} x_{4} x_{5} x_{1}$ be a 5 -cycle in $G$. Then, since $G$ is cubic and has girth $5, N^{\prime}(C)$ must contain exactly five vertices.

Suppose first that $N^{\prime}(C)$ contains a cycle $y_{1} y_{2} y_{3} y_{4} y_{5} y_{1}$. Then without loss of generality, we may suppose that $y_{1} \sim x_{1}, y_{2} \sim x_{3}, y_{3} \sim x_{5}, y_{4} \sim x_{2}$ and $y_{5} \sim x_{4}$. But then $G \cong P_{10}$.

Suppose next that $N^{\prime}(C)$ contains a path of length 4 which we denote by $y_{1} y_{2} y_{3} y_{4} y_{5}$. Again, without loss of generality, we may suppose that $y_{i} \sim x_{i}$, for $i=1, \ldots, 5$. But now if $y_{1} \nsim y_{5},\left\{y_{1}, y_{5}\right\}$ is a 2 -cut in $G$, a contradiction. Hence $y_{1} \sim y_{5}$ and again $G \cong P_{10}$.

Finally, suppose $N^{\prime}(C)$ contains a 3 -path which we will denote by $y_{1} y_{2} y_{3} y_{4}$. As before, we may suppose that $y_{1} \sim x_{1}, y_{2} \sim x_{3}, y_{3} \sim x_{5}$ and $y_{4} \sim x_{2}$. Since $G$ is cubic, there must then exist a fifth vertex $y_{5} \in N^{\prime}(C)$ such that $y_{5} \sim x_{4}$. Now also since $G$ is cubic, there must exist a vertex $z \in V(G), z \neq x_{1}, \ldots, x_{5}, y_{1}, \ldots, y_{5}$. By 3 -connectivity and Menger's theorem, there must be three paths in $G$ joining $z$ to vertices $y_{1}, y_{4}$ and $y_{5}$ respectively. In other words, there must exist a bridge (containing vertex $z$ ) with vertices of attachment $y_{1}, y_{4}$ and $y_{5}$ in $C \cup N^{\prime}(C)$. Hence, in particular, vertices $x_{1}$ and $x_{4}$ are a co-bridge pair. But by Lemma 2.1, these two vertices are a non-co-bridge pair and we have a contradiction.

Next suppose $N^{\prime}(C)$ contains a path of length 2. Elimination of this case will be the culmination of the next two lemmas.

Lemma 3.2: Let $G$ be a cubic 3 -connected graph of girth 5 such that all odd cycles of length greater than 5 have a chord. Then if $G$ contains a subgraph isomorphic to graph $J_{1}$ shown in Figure $3.1, G \cong P_{10}$. 

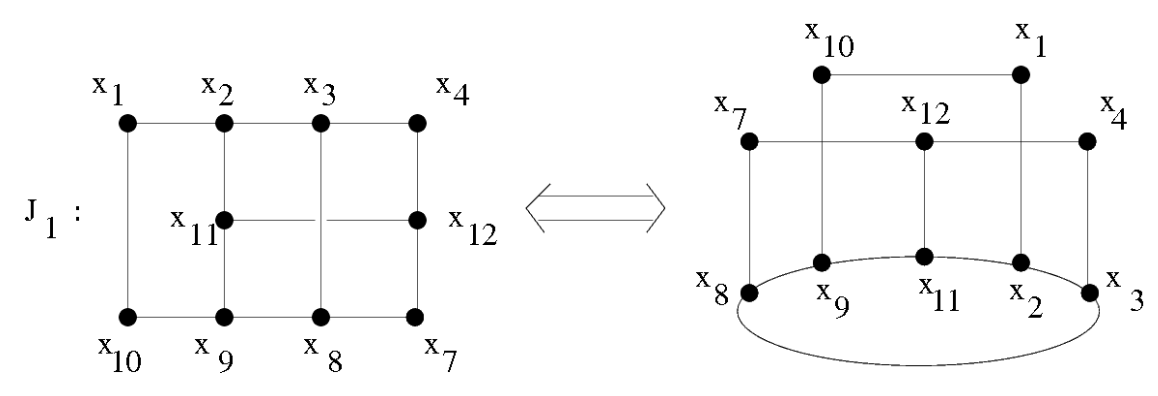

Figure 3.1

Proof: Suppose $G \neq P_{10}$, but $G$ does contain as a subgraph the graph $J_{1}$. We adopt the vertex labeling of Figure 3.1.

Claim 1: The subgraph $J_{1}$ must be induced.

It is easy to check that adding any edge different from $x_{1} x_{7}$ and $x_{4} x_{10}$ results in the formation of a cycle of size less than five, contradicting the girth hypothesis.

So then let us assume $x_{1}$ is adjacent to $x_{7}$. Then if $C=x_{2} x_{3} x_{8} x_{9} x_{11} x_{2}, N^{\prime}(C)$ contains the induced path $x_{10} x_{1} x_{7} x_{12} x_{4}$ of length 4, contradicting Lemma 3.1. By symmetry, if we add the edge $x_{4} x_{10}$, a similar contradiction is reached. This proves Claim 1.

Claim 2: For $1 \leq i<j \leq 12, N_{i}^{\prime} \cap N_{j}^{\prime}=\emptyset$.

It is routine to check that any possible non-empty intersection of two different $N_{i}^{\prime} \mathrm{s}$ produces either a cycle of length less than 5 , thus contradicting the girth hypothesis, or else a 7-cycle, thus contradicting Lemma 2.3. This proves Claim 2.

Claim 3: For $(i, j) \in\{(1,4),(1,7),(4,10),(7,10)\}$, there is no edge joining $N_{i}^{\prime}$ and $N_{j}^{\prime}$.

This is immediate by Lemma 2.3 .

For $i=1,4,7,10$, let $y_{i}$ denote the (unique) neighbor of $x_{i}$ which does not lie in $J_{1}$.

Then since $G$ is cubic and 3-connected, there must be a bridge $B$ in $G-V\left(J_{1}\right)$ with at least three vertices of attachment from the set $\left\{y_{1}, y_{4}, y_{7}, y_{10}\right\}$. It then follows that either $\left\{x_{1}, x_{7}\right\}$ or $\left\{x_{4}, x_{10}\right\}$ is a co-bridge pair. But these pairs are both well-connected and hence by Lemma 2.1, neither is a co-bridge pair, a contradiction.

Lemma 3.3: Let $G$ be a cubic 3-connected graph of girth 5 such that all odd cycles of length greater than 5 have a chord. Suppose $C$ is a girth cycle in $G$ such that $N^{\prime}(C)$ contains a path of length 2. (That is, $G$ contains a subgraph isomorphic to graph $J_{2}$ shown in Figure 3.2.) Then $G \cong P_{10}$. 


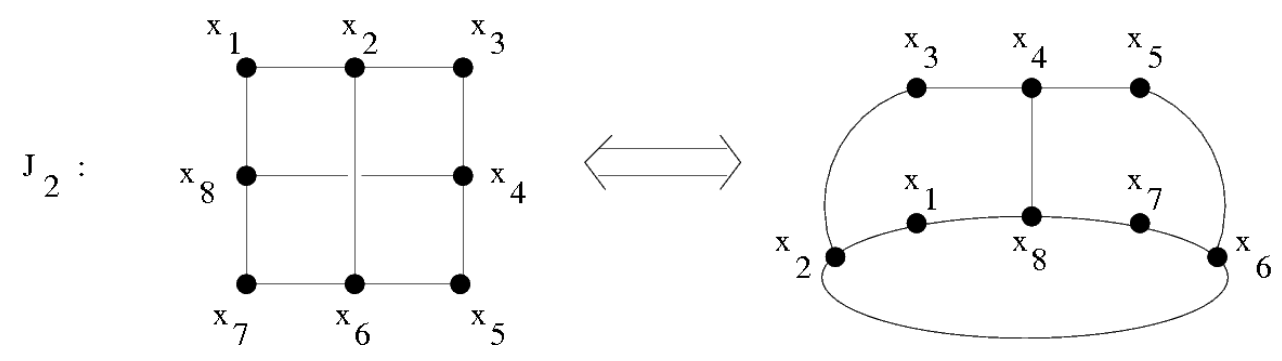

Figure 3.2

Proof: Suppose $G \approx P_{10}$, but $G$ does contain a subgraph isomorphic to $J_{2}$. We adopt the vertex labeling shown in Figure 3.2.

Claim 1: $J_{2}$ is an induced subgraph.

This is immediate via the girth 5 hypothesis.

Claim 2: For $1 \leq i<j \leq 8, N_{i}^{\prime} \cap N_{j}^{\prime}=\emptyset$. For all pairs $\{i, j\} \neq\{1,5\}$ and $\{3,7\}$, this follows from the girth 5 hypothesis and observing that $N_{2}^{\prime}=N_{4}^{\prime}=N_{6}^{\prime}=N_{8}^{\prime}=\emptyset$. Suppose, then, that there exists a vertex $y \in N_{1}^{\prime} \cap N_{5}^{\prime}$. Then if we let $C=x_{1} x_{2} x_{6} x_{7} x_{8} x_{1}$ we find that $N^{\prime}(C)$ contains a path $y x_{5} x_{4} x_{3}$ of length 3, contradicting Lemma 3.1 .

So $N_{1}^{\prime} \cap N_{5}^{\prime}=\emptyset$ and by symmetry, $N_{3}^{\prime} \cap N_{7}^{\prime}=\emptyset$ as well. This proves Claim 2 .

For $i=1,3,5,7$, let $y_{i}$ be the neighbor of $x_{i}$ not in $J_{2}$.

Claim 3: For $\{i, j\} \in\{\{1,3\},\{1,5\},\{1,7\},\{3,5\},\{3,7\},\{5,7\}\}$, there is no edge joining $y_{i}$ and $y_{j}$.

By symmetry, we need only check the pairs $\{1,3\}$ and $\{1,5\}$. If there is an edge joining $y_{1}$ and $y_{3}$, there is then a subgraph isomorphic to $J_{1}$ and we are done by Lemma 3.2. If, on the other hand, $y_{1} \sim y_{5}$, we have a 7-cycle in $G$, contradicting Lemma 2.3. This proves Claim 3.

It is easily checked that $\left\{x_{1}, x_{5}\right\}$ and $\left\{x_{3}, x_{7}\right\}$ are each well-connected and hence by Claim 3 and Lemma 2.1 each is a non-co-bridge pair. On the other hand, since $G$ is cubic and 3 -connected, there is a bridge $B$ of the subgraph spanned by $V\left(J_{2}\right) \cup\left\{y_{1}, y_{3}, y_{5}, y_{7}\right\}$ which must have attachments at at least three of the vertices $\left\{y_{1}, y_{3}, y_{5}, y_{7}\right\}$. But it then follows that either $\left\{x_{1}, x_{5}\right\}$ or $\left\{x_{3}, x_{7}\right\}$ is a co-bridge pair, a contradiction.

The next two results culminate in the elimination of the case in which there is a matching of size 2 in $N^{\prime}(C)$.

Lemma 3.4: Suppose $G$ is a cubic 3-connected graph of girth 5 in which every odd cycle of length greater than 5 has a chord. Suppose $G$ contains the graph $L_{1}$ shown in Figure 3.3 as a subgraph. Then $G \cong P_{10}$. 

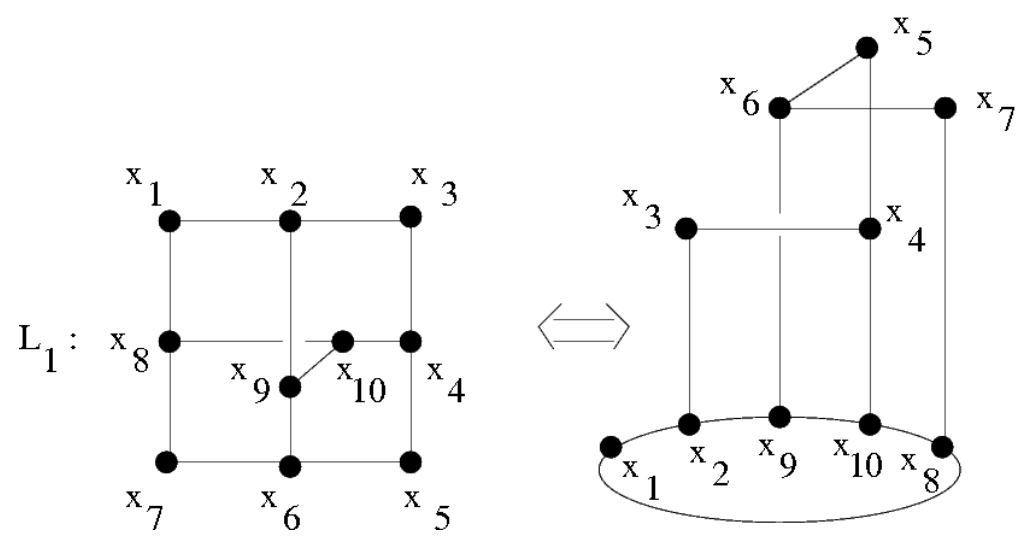

\section{Figure 3.3}

Proof: Suppose $G ¥ P_{10}$, but suppose $G$ does contain the graph $L_{1}$ as a subgraph. We assume the vertices of this subgraph $L_{1}$ are labeled as in Figure 3.3.

Claim 1: $L_{1}$ is an induced subgraph.

By the girth hypothesis, if vertices $x_{i}$ and $x_{j}$ are joined by a path of length at most 3 , then they are not adjacent. Therefore, by symmetry we need check only the pairs $\left\{x_{1}, x_{5}\right\}$ and $\left\{x_{3}, x_{7}\right\}$. However, if $x_{1} \sim x_{5}, L_{1} \cup x_{1} x_{5} \cong P_{10} \backslash e$. But this graph contains a girth cycle $C$ such that $N^{\prime}(C)$ contains a path of length 3 and so by Lemma 3.1, $G \cong P_{10}$, a contradiction. So $x_{1} \nsim x_{5}$. By symmetry, $x_{3} \nsim x_{7}$ as well and Claim 1 is proved.

Since $G$ is cubic, $N_{2}^{\prime}=N_{4}^{\prime}=N_{6}^{\prime}=N_{8}^{\prime}=N_{9}^{\prime}=N_{10}^{\prime}=\emptyset$ and each of $N_{1}^{\prime}, N_{3}^{\prime}, N_{5}^{\prime}$ and $N_{7}^{\prime}$ consists of a single vertex. Let $N_{i}^{\prime}=\left\{y_{i}\right\}$ for $i=1,3,5,7$ and let $L_{1}^{\prime}=\left\{y_{1}, y_{3}, y_{5}, y_{7}\right\}$.

Claim 2: $y_{1}, y_{3}, y_{5}$ and $y_{7}$ are all distinct.

By symmetry, we need only check that $y_{1} \neq y_{3}$ and $y_{1} \neq y_{5}$. The first of these assertions follows immediately via the girth hypothesis. If $y_{1}=y_{5}$, on the other hand, it follows that $y_{1} x_{1} x_{2} x_{9} x_{10} x_{4} x_{5} y_{5}\left(=y_{1}\right)$ is a chordless 7-cycle, contrary to hypothesis. Thus Claim 2 is true.

Claim 3: $L_{1}^{\prime}$ is independent.

Indeed, if there were an edge joining any two vertices of $L_{1}^{\prime}$, one can find a chordless 7 -cycle containing it, which is a contradiction.

Since $G$ is cubic, $G-\left(L_{1} \cup L_{1}^{\prime}\right) \neq \emptyset$. Therefore, there must exist a non-edge bridge $B$ with attachments on at least three of $y_{1}, y_{3}, y_{5}$ and $y_{7}$. But then $B$ must have either both $y_{1}$ and $y_{5}$ as vertices of attachment or both $y_{3}$ and $y_{7}$ as vertices of attachment. By symmetry, without loss of generality, let us assume that $y_{1}$ and $y_{5}$ are vertices of attachment for $B$. Hence $x_{1}$ and $x_{5}$ are a co-bridge pair. 
On the other hand, the induced paths $x_{1} x_{2} x_{3} x_{4} x_{5}$ and $x_{1} x_{2} x_{9} x_{10} x_{4} x_{5}$ serve to show that $x_{1}$ and $x_{5}$ are well-connected, and since there does not exist an edge joining $y_{1}$ and $y_{5}$, it follows from Lemma 2.1 that $\left\{x_{1}, x_{5}\right\}$ is a non-co-bridge pair. Hence we have a contradiction and Lemma 3.4 is proved.

Lemma 3.5: Suppose $G$ is a cubic 3-connected graph of girth 5 in which every odd cycle of length greater than 5 has a chord. Suppose $G$ contains the graph $L_{2}$ shown in Figure 3.4 as a subgraph. Then $G \cong P_{10}$.

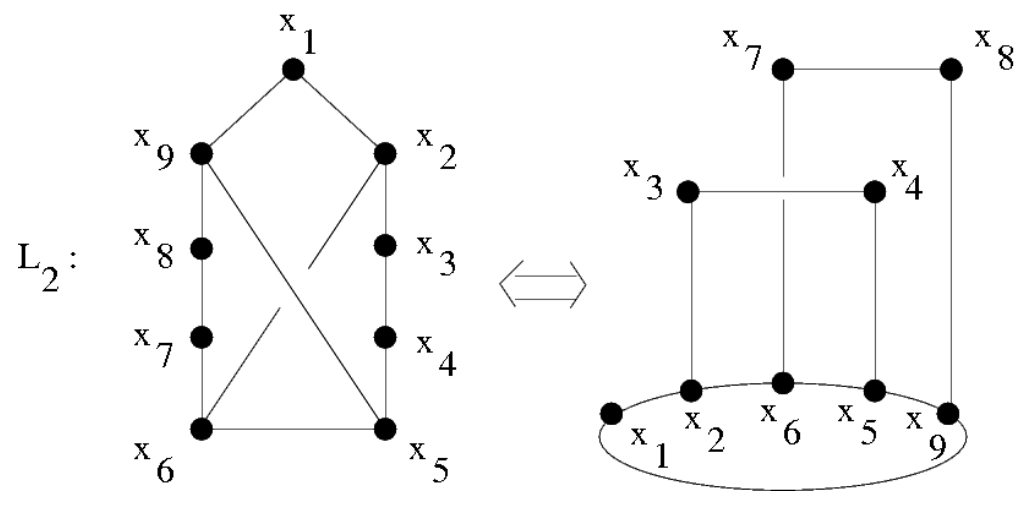

Figure 3.4

Proof: Suppose $G ¥ P_{10}$, but suppose $G$ does contain the graph $L_{2}$ as a subgraph. We assume the vertices of this subgraph $L_{2}$ are as labeled in Figure 3.4.

Claim 1: $L_{2}$ is an induced subgraph of $G$.

As before, we need only check pairs of vertices $\left\{x_{i}, x_{j}\right\}$ which lie at distance at least 4. Hence we need only check the pair $\left\{x_{3}, x_{8}\right\}$. If $x_{3}$ and $x_{8}$ are joined by an edge, then the resulting graph is isomorphic to $P_{10} \backslash v$. But this graph contains a girth cycle $C$ such that $N^{\prime}(C)$ contains a path of length 3 and so by Lemma 3.1, $G \cong P_{10}$, a contradiction. Hence $x_{3} \nsim x_{8}$ and Claim 1 is true.

Since $G$ is cubic, $N_{2}^{\prime}=N_{5}^{\prime}=N_{6}^{\prime}=N_{9}^{\prime}=\emptyset$ and each of $N_{1}^{\prime}, N_{3}^{\prime}, N_{4}^{\prime}, N_{7}^{\prime}$ and $N_{8}^{\prime}$ consists of a single vertex. Let $N_{i}^{\prime}=\left\{y_{i}\right\}$ for $i=1,3,4,7,8$ and let $L_{2}^{\prime}=\left\{y_{1}, y_{3}, y_{4}, y_{7}, y_{8}\right\}$.

Claim 2: $y_{1}, y_{3}, y_{4}, y_{7}$ and $y_{8}$ are all distinct.

By the girth hypothesis, we need only check that $y_{i} \neq y_{j}$ when $x_{i}$ and $x_{j}$ are at distance at least 3 . By symmetry, then, we need only check the five pairs $\left\{y_{1}, y_{4}\right\},\left\{y_{3}, y_{7}\right\}$, $\left\{y_{3}, y_{8}\right\},\left\{y_{4}, y_{7}\right\}$ and $\left\{y_{4}, y_{8}\right\}$.

Suppose $y_{1}=y_{4}$. Then consider the 5-cycle $C=x_{1} x_{2} x_{6} x_{5} x_{9} x_{1}$ and note that $N^{\prime}(C)$ contains the path $y_{1}\left(=y_{4}\right) x_{4} x_{3}$ and by the girth hypothesis, this is an induced path of length 2 . But then by Lemma 3.3, $G \cong P_{10}$, a contradiction. Hence $y_{1} \neq y_{4}$.

If $y_{3}=y_{7}, y_{3} x_{3} x_{4} x_{5} x_{9} x_{8} x_{7} y_{7}\left(=y_{3}\right)$ is a chordless 7 -cycle, a contradiction. If $y_{3}=y_{8}, y_{3} x_{3} x_{4} x_{5} x_{6} x_{7} x_{8} y_{8}\left(=y_{3}\right)$ is a chordless 7 -cycle, a contradiction. Suppose next 
that $y_{4}=y_{8}$. Then the 10-vertex subgraph $L_{2} \cup\left\{x_{4} y_{4}, y_{4} x_{7}\right\}$ is isomorphic to $L_{1}$ and hence by Lemma $3.4, G \cong P_{10}$, a contradiction.

Finally, if $y_{4}=y_{8}, y_{4} x_{4} x_{3} x_{2} x_{1} x_{9} x_{8} y_{8}\left(=y_{4}\right)$ is a chordless 7 -cycle, a contradiction. Thus Claim 2 is true.

Claim 3: For $i, j \in\{1,3,4,7,8\}$, there is no edge joining $y_{i}$ and $y_{j}$, that is, $L_{2}^{\prime}$ is independent.

By the girth 5 hypothesis and symmetry, we need only check the pairs $\{i, j\}=$ $\{1,3\},\{1,4\},\{3,7\},\{3,8\},\{4,7\}$. But if there is an edge joining $y_{1}$ and $y_{3}$, using induced path $x_{1} x_{9} x_{5} x_{4} x_{3}$ we obtain a chordless 7 -cycle, a contradiction. Similarly, for the pair $\{1,4\}$ using induced path $x_{1} x_{2} x_{6} x_{5} x_{4}$, for $\{3,7\}$, using induced path $x_{3} x_{4} x_{5} x_{6} x_{7}$, for $\{3,8\}$, using induced path $x_{3} x_{4} x_{5} x_{9} x_{8}$, and for $\{4,7\}$, using induced path $x_{4} x_{3} x_{2} x_{6} x_{7}$, we obtain a chordless 7 -cycle, a contradiction in each case. This proves Claim 3.

Since $G$ is cubic, $G-\left(L_{2} \cup L_{2}^{\prime}\right) \neq \emptyset$. Therefore, there must exist a non-edge bridge $B$ with attachments on at least three of $y_{1}, y_{3}, y_{4}, y_{7}$ and $y_{8}$.

First assume that there is attachment at vertex $y_{1}$. Vertices $x_{1}$ and $x_{3}$ are wellconnected using induced paths $x_{1} x_{2} x_{3}$ and $x_{1} x_{9} x_{5} x_{6} x_{2} x_{3}$ and since $x_{1} \nsim x_{3},\left\{x_{1}, x_{3}\right\}$ is a non-co-bridge pair by Lemma 2.1. Hence $B$ has no attachment at vertex $y_{3}$.

Similarly, paths $x_{1} x_{2} x_{3} x_{4}$ and $x_{1} x_{2} x_{6} x_{5} x_{4}$ serve to show that $x_{1}$ and $x_{4}$ are wellconnected and hence $\left\{x_{1}, x_{4}\right\}$ is a non-co-bridge pair as well. Hence there is no attachment for $B$ at $y_{4}$. By symmetry, there is no attachment for $B$ at $y_{7}$ or $y_{8}$ either. Thus there is no attachment at $y_{1}$.

So $B$ must have attachments at at least three of the four vertices $y_{3}, y_{4}, y_{7}$ and $y_{8}$. By symmetry, it is enough to consider the possibilities of attachments at $y_{3}, y_{4}$ and $y_{7}$ or at $y_{3}, y_{4}$ and $y_{8}$. But in the former case, induced paths $x_{3} x_{2} x_{6} x_{7}$ and $x_{3} x_{4} x_{5} x_{6} x_{7}$ serve to show that $x_{3}$ and $x_{7}$ are well-connected and since they are not adjacent, by Lemma $2.1\left\{x_{3}, x_{7}\right\}$ is a non-co-bridge pair. Similarly, in the latter case, induced paths $x_{3} x_{4} x_{5} x_{6} x_{7} x_{8}$ and $x_{3} x_{2} x_{1} x_{9} x_{8}$ suffice to show that $x_{3}$ and $x_{8}$ are also well-connected and hence $\left\{x_{3}, x_{8}\right\}$ is a non-co-bridge pair as well. Thus we have a contradiction and the proof of Lemma 3.5 is complete.

Finally, we treat the case when there is a single edge in $N^{\prime}(C)$.

Lemma 3.6: Suppose $G$ is a cubic 3-connected graph of girth 5 in which every odd cycle of length greater than 5 has a chord. Suppose $G$ contains the graph $L_{3}$ shown in Figure 3.5 as a subgraph. Then $G \cong P_{10}$. 

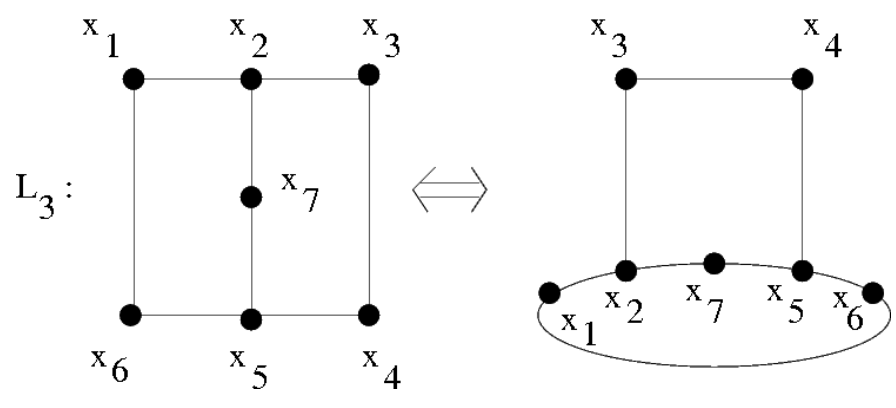

\section{Figure 3.5}

Proof: Suppose $G \neq P_{10}$, but suppose $G$ does contain the graph $L_{3}$ as a subgraph.

Claim 1: $L_{3}$ is induced.

This follows immediately from the girth hypothesis, since the diameter of $L_{3}$ is only 3.

Since $G$ is cubic, $N_{2}^{\prime}=N_{5}^{\prime}=\emptyset$ and each of $N_{1}^{\prime}, N_{3}^{\prime}, N_{4}^{\prime}, N_{6}^{\prime}$ and $N_{7}^{\prime}$ consists of a single vertex. Let $N_{i}^{\prime}=\left\{y_{i}\right\}$, for $i=1,3,4,6,7$ and let $L_{3}^{\prime}=\left\{y_{1}, y_{3}, y_{4}, y_{6}, y_{7}\right\}$.

Claim 2: $y_{1}, y_{3}, y_{4}, y_{6}$ and $y_{7}$ are all distinct.

By the girth hypothesis, we need only check pairs at distance at least 3 and hence we need only check $\left\{x_{1}, x_{4}\right\}$.

Suppose $y_{1}=y_{4}$. Then consider the 5-cycle $C=x_{1} x_{8} x_{4} x_{5} x_{6} x_{1}$ and note that $N^{\prime}(C)$ contains the 2-path $x_{3} x_{2} x_{7}$ and moreover, this 2-path is induced by the girth hypothesis. Hence by Lemma $3.3, G \cong P_{10}$, a contradiction. Hence $y_{1} \neq y_{4}$ and Claim 2 is proved.

Claim 3: For $i, j \in\{1,3,4,6,7\}$, there is no edge joining $y_{i}$ and $y_{j}$, that is, $L_{3}^{\prime}$ is independent.

By symmetry, we need only check the pairs $\{i, j\}=\{1,3\},\{1,4\}$ and $\{1,7\}$. But if there is an edge joining $y_{1}$ and $y_{3}$, using induced path $x_{1} x_{6} x_{5} x_{4} x_{3}$ we obtain a chordless 7 -cycle, a contradiction. Similarly, for the pair $\{1,4\}$, using induced path $x_{1} x_{2} x_{7} x_{5} x_{4}$ we obtain a chordless 7 -cycle as well.

Suppose, then, that $y_{1} \sim y_{7}$ and consider $L_{3} \cup\left\{x_{1} y_{1}, y_{1} y_{7}, y_{7} x_{7}\right\}$. The 5-cycle $C=x_{1} x_{2} x_{7} x_{5} x_{6} x_{1}$ has the property that $N^{\prime}(C)$ contains the independent edges $y_{1} y_{7}$ and $x_{3} x_{4}$. But then by Lemma 3.5, $G \cong P_{10}$, a contradiction, and Claim 3 is proved.

Claim 4: The pairs $\left\{x_{i}, x_{j}\right\}$, for $\{i, j\}=\{1,4\},\{1,7\},\{3,6\},\{3,7\},\{4,7\}$ and $\{6,7\}$ are well-connected.

By symmetry, it suffices to treat only the two pairs $\{1,4\}$ and $\{1,7\}$. But induced paths $x_{1} x_{2} x_{3} x_{4}$ and $x_{1} x_{2} x_{7} x_{5} x_{4}$ show that $\left\{x_{1}, x_{4}\right\}$ is well-connected, while for $\left\{x_{1}, x_{7}\right\}$, paths $x_{1} x_{2} x_{7}$ and $x_{1} x_{6} x_{5} x_{7}$ guarantee well-connectedness. 
Claim 5: Each of $\left\{x_{1}, x_{4}\right\},\left\{x_{1}, x_{7}\right\},\left\{x_{3}, x_{6}\right\},\left\{x_{3} x_{7}\right\},\left\{x_{4}, x_{7}\right\}$ and $\left\{x_{6}, x_{7}\right\}$ is a non-cobridge pair.

These are all pairs of non-adjacent vertices and hence by Lemma 2.1, the Claim follows.

Since $G$ is cubic, there must be a bridge $B$ in $V(G)-\left(L_{3} \cup L_{3}^{\prime}\right)$. Since $G$ is 3 -connected, bridge $B$ must then have three vertices of attachment among the set $\left\{y_{1}, y_{3}, y_{4}, y_{6}, y_{7}\right\}$. Vertex $y_{7}$ cannot be one of the three, since $\left\{x_{i}, x_{7}\right\}$ is a non-cobridge pair, for $i=1,3,4$ and 6 , by Claim 5 . It then follows that either $\left\{y_{1}, y_{4}\right\}$ or $\left\{y_{3}, y_{6}\right\}$ are attachment sets for $B$. But these pairs are non-co-bridge pairs by Claim 5 and we have a contradiction. The Lemma follows.

We are now prepared for our main result for cubic graphs.

Theorem 3.7: Suppose $G$ is a cubic 3-connected graph of girth 5 in which every odd cycle of length greater than 5 has a chord. Then $G \cong P_{10}$.

Proof: Let $C$ be a 5-cycle in $G$. By Lemmas 3.1, 3.3, 3.5 and 3.6, we may assume that $N^{\prime}(C)$ is independent. So once again, since $G$ is cubic, there must be a bridge in $G-\left(V(C) \cup N^{\prime}(C)\right)$ with vertices of attachments in at least three of $N_{1}^{\prime}, \ldots, N_{5}^{\prime}$. Hence there must be two non-adjacent $x_{i}$ 's which are a co-bridge pair. But this contradicts Lemma 2.1 and the Theorem is proved.

The original conjecture of Robertson did not include the assumption that the graphs are internally-4-connected. However, without this assumption, the conclusion does not follow as is shown by the following counterexample.

Let $G_{1}$ be the bipartite graph on twenty-six vertices and $G_{2}$, the graph on twelve vertices shown in Figure 3.6.

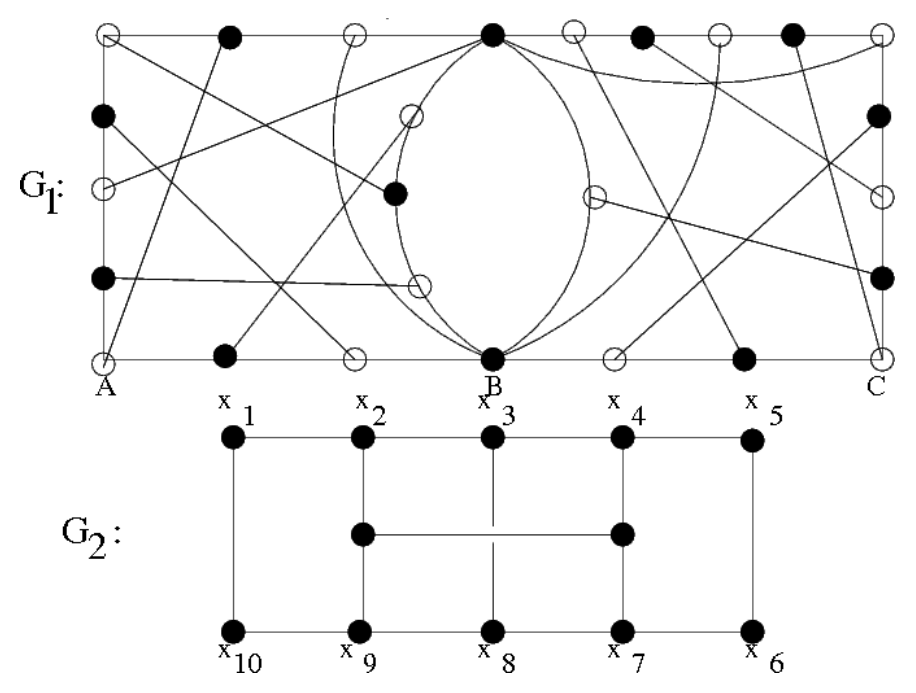

Figure 3.6 
Join a copy of $G_{1}$ to one central copy of $G_{2}$ by joining $A$ to $x_{1}, B$ to $x_{2}$ and $C$ to $x_{3}$ via a matching, a second copy of $G_{1}$ to $G_{2}$ by joining $A$ to $x_{3}, B$ to $x_{4}$ and $C$ to $x_{5}$ via a matching, a third copy of $G_{1}$ to $G_{2}$ by joining $A$ to $x_{6}, B$ to $x_{7}$ and $C$ to $x_{8}$ via a matching, and a fourth copy of $G_{1}$ to $G_{2}$ by joining $A$ to $x_{8}, B$ to $x_{9}$ and $C$ to $x_{10}$ via a matching. The resulting graph on 116 vertices is 3 -connected, has girth 5 and every odd cycle of length greater than 5 has a chord. Clearly, it is not internally 4-connected.

Note that we may obtain infinitely many more counterexamples by attaching additional copies of the graph $G_{1}$ to each other along their common path $A \cdots B \cdots C$ shown in Figure 3.6.

\section{4. $N^{\prime}(C)$ is not independent.}

Beginning in this section we turn our attention to the original conjecture in which we drop the assumption that $G$ is cubic, but add the assumption that $G$ is internally-4connected. In these next three sections, we will follow, as far as we can, the general approach of Section 3 in that we will begin with a 5 -cycle $C$ and analyze the structure of the subgraph induced by $N^{\prime}(C)$. In doing so, we will see that a number of claims follow just as they did in the cubic case. But not all.

Lemma 4.1: Let $G$ be a 3-connected internally 4-connected graph of girth 5 in which every odd cycle of length greater than 5 has a chord. Then if $C$ is a 5 -cycle in $G, N^{\prime}(C)$ is not an independent set.

Proof. By way of contradiction, let us suppose that $C=x_{1} x_{2} x_{3} x_{4} x_{5} x_{1}$ is a 5 -cycle in $G$ such that $N^{\prime}(C)$ is independent. Note that by the girth 5 hypothesis, $N_{i}^{\prime} \cap N_{j}^{\prime}=\emptyset$, for $1 \leq i<j \leq 5$. Note also that since $N^{\prime}(C)$ is independent, there exist no edge-bridges of $C \cup N^{\prime}(C)$.

For any vertex pair $\left\{x_{i}, x_{i+2}\right\}$ in $V(C)$, (where $i$ is read modulo 5), consider the set $V(C)-\left\{x_{i}, x_{i+2}\right\}$ which separates the vertices $x_{i}$ and $x_{i+2}$ on $C$. Since $x_{i}$ and $x_{i+2}$ are well-connected in $C$, by Lemma 2.1 they form a non-co-bridge pair. Similarly, any non-adjacent pair of vertices $x_{i}$ and $x_{j}$ on $C$ are a non-co-bridge pair. In fact, then, all non-edge bridges are either monobridges or bibridges with one attachment in some $N_{i}^{\prime}$ and another in $N_{i+1}^{\prime}$, for some $i,(\bmod 5)$.

Since $G$ is internally 4 -connected, there must be a third $x_{i}-x_{i+2}$ (induced) path $P_{i, i+2}$ containing none of the vertices $V(C)-\left\{x_{i}, x_{i+2}\right\}$. Such a path must visit $N_{i}^{\prime}$ and $N_{i+2}^{\prime}$. More particularly, this path can be assumed to visit, in turn, a sequence of sets of the form $\left\{x_{i}\right\}, N_{i}^{\prime}, B_{i, i+1}, N_{i+1}^{\prime}, B_{i+1, i+2}, N_{i+2}^{\prime},\left\{x_{i+2}\right\}$, or else, going around cycle $C$ in the opposite direction, a sequence of sets of the form $\left\{x_{i}\right\}, B_{i, i+4}, N_{i+4}^{\prime}, B_{i+4, i+3}, N_{i+3}^{\prime}$, $B_{i+3, i+2}, N_{i+2}^{\prime},\left\{x_{i+2}\right\}$. In the first instance above, the path is called a short overpath and in the second, a long overpath. Note that both long and short overpaths may use monobridges.

(In Figure 4.1, $P_{1,3}$ denotes a short $\left\{x_{1}, x_{3}\right\}$ overpath and in Figure $4.2, Q_{3,1}$ is a long $\left\{x_{3}, x_{1}\right\}$ overpath.) 


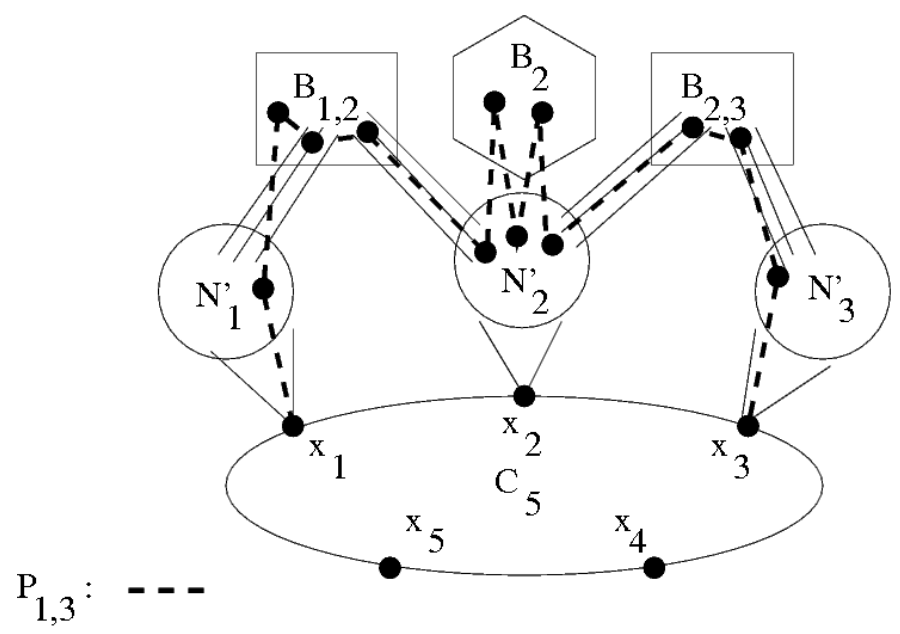

Figure 4.1. A short overpath.

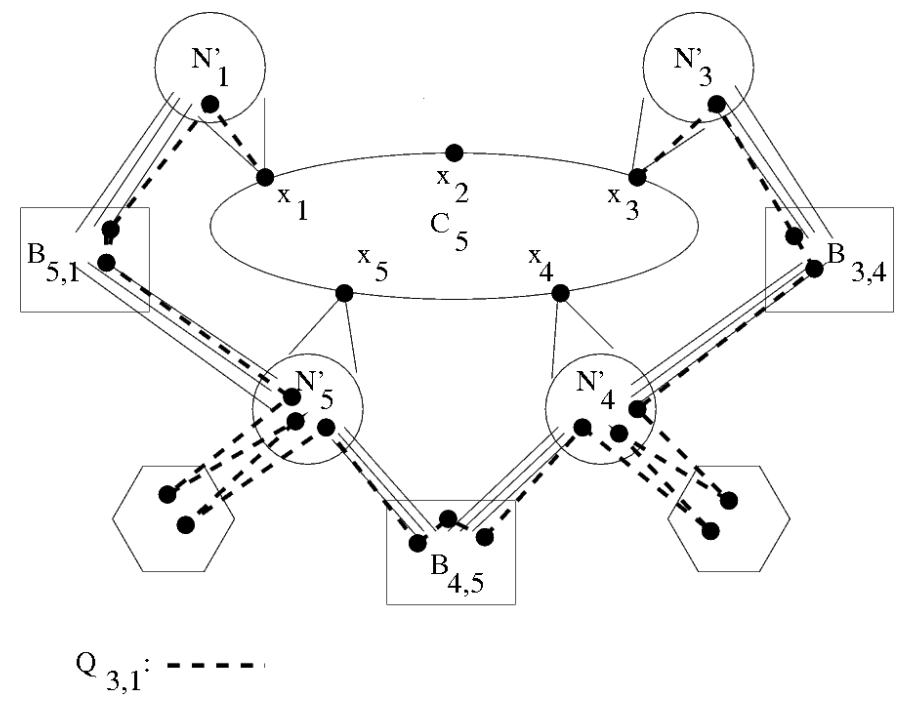

Figure 4.2. A long overpath.

Note that, if there is a short overpath of even length, or a long overpath of odd length, it can be taken together with a subpath of $C$ of suitable parity to form a chordless odd cycle of length greater than 5 , contrary to hypothesis. So we have the next observation.

(1) Every short overpath is of odd length and every long overpath is of even length.

We may also assume the following.

(2) Any pair of type $\left\{x_{i}, x_{i+2}\right\}(\bmod 5)$ cannot be joined by both a short and a long overpath.

To see this, suppose, to the contrary, that some pair is joined by both a short overpath $P$ and a long overpath $Q$. Then $P \cup Q$ would contain an odd cycle of length 
greater than 5 (in fact, at least 9), containing no chords, contradicting an hypothesis of this lemma.

Henceforth, therefore, we shall say the pair $\left\{x_{i}, x_{i+2}\right\}$ is short (respectively, long) if it is joined by a short (respectively, long) overpath.

We also claim the following is true.

(3) There cannot exist simultaneously a short $\left\{x_{i}, x_{i+2}\right\}$ overpath and a short $\left\{x_{i+2}\right.$, $\left.x_{i+4}\right\}$ overpath.

To see this, suppose that both short overpaths exist. Since by (1), both are of odd length, together with the single edge $x_{i+4} x_{i}$, their union contains a chordless odd cycle of length greater than 5, again a contradiction.

The next is an obvious observation.

(4) Any long $\left\{x_{i}, x_{i+3}\right\}$ overpath gives rise to both a short $\left\{x_{i}, x_{i+2}\right\}$ overpath and a short $\left\{x_{i+1}, x_{i+3}\right\}$ overpath via a suitable selection of an edge joining $x_{i+1}$ and $N_{i+1}^{\prime}$ and an edge joining $x_{i+2}$ and $N_{i+2}^{\prime}$.

(5) For some choice of $i$, there must exist a short $\left\{x_{i}, x_{i+2}\right\}$ overpath.

For suppose, to the contrary, that, for all $i=1, \ldots, 5$, no short $\left\{x_{i}, x_{i+2}\right\}$ overpath exists. Fix $i$. Then by internal-4-connectivity, a long $\left\{x_{i+2}, x_{i}\right\}$ overpath $P$ must exist. But then we are done by (4).

Without loss of generality, then, let us suppose that $\left\{x_{1}, x_{3}\right\}$ is short. Then by (3) $\left\{x_{3}, x_{5}\right\}$ cannot be short and hence must be long. Again by (3) and symmetry, $\left\{x_{4}, x_{1}\right\}$ cannot be short, and hence must be long as well. Thus by (4), $\left\{x_{2}, x_{5}\right\}$ must be short. Hence by (3), $\left\{x_{2}, x_{4}\right\}$ is long. By (4), then, $\left\{x_{1}, x_{4}\right\}$ is short and we have a contradiction. This proves the lemma. 


\section{Forbidden induced paths of length at least 3.}

Again let us suppose that $G$ is a graph which is 3-connected, has girth 5 and that $G$ has the further property that every odd cycle of length greater than 5 has a chord. This entire section is devoted to showing that if $C$ is a 5-cycle in $G$ and $N^{\prime}(C)$ contains an induced path of length at least 3 , then $G \cong P_{10}$. Note that we do not use the internal-4connected assumption in this section. Hence Lemma 3.1 for cubic graphs is a corollary of Lemma 5.4. However, we have chosen to include the direct proof of Lemma 3.1 given in Section 3 to give the reader more appreciation as to how much the cubic assumption simplifies matters.

Lemma 5.1: Suppose $G$ is 3-connected, has girth 5 and all odd cycles of length greater than 5 have a chord. Let $C_{1}=x_{1} x_{2} x_{3} x_{4} x_{5} x_{1}$ be a cycle of length 5 in $G$ and let $C_{2}=y_{1} y_{2} \cdots y_{5} \cdots$ be any cycle in $N^{\prime}\left(C_{1}\right)$. Then $\left|C_{2}\right|=5, G\left[C_{1} \cup C_{2}\right] \cong P_{10}$ and $N^{\prime}\left(C_{1}\right) \backslash V\left(C_{2}\right)$ is independent.

Proof: Let $C_{1}=x_{1} x_{2} x_{3} x_{4} x_{5} x_{1}$ and let $C_{2}=y_{1} y_{2} \cdots y_{k} y_{1}$. Suppose $k>5$. Without loss of generality, assume that $x_{1} \sim y_{1}$. Then by symmetry, we may assume that $y_{2} \sim x_{3}$. But then it follows that $y_{3} \sim x_{5}, y_{4} \sim x_{2}$ and $y_{5} \sim x_{4}$. If $y_{6}$ is adjacent to any of $x_{2}, \ldots, x_{5}$, the girth 5 hypothesis is contradicted. But then $y_{6} \sim x_{1}$. So $x_{1} y_{1} y_{2} x_{3} x_{4} y_{5} y_{6} x_{1}$ is a 7 -cycle in $G$, a contradiction of Lemma 2.3. Therefore $C_{2}$ is a 5-cycle. Thus $G\left[C_{1} \cup C_{2}\right] \cong P_{10}$ as claimed.

Now by way of contradiction, let us suppose that $N^{\prime}\left(C_{1}\right) \backslash V\left(C_{2}\right)$ contains an edge $y_{6} y_{7}$. By the symmetry of the Petersen graph, we may then suppose, without loss of generality, that $y_{6} x_{5} \in E(G)$. Then since the girth of $G$ is $5, y_{7} \nsim x_{5}, y_{7} \nsim x_{4}$ and $y_{7} \nsim x_{1}$. If $y_{7} \sim x_{2}$, then $x_{2} y_{4} y_{5} x_{4} x_{5} y_{6} y_{7} x_{2}$ is a 7-cycle, contradicting Lemma 2.3. So $y_{7} \nsim x_{2}$. Similarly, if $y_{7} \sim x_{3}$, then we get a 7 -cycle $y_{7} y_{6} x_{5} y_{3} y_{4} x_{2} x_{3} y_{7}$, a contradiction, so $y_{7} \nsim x_{3}$. Thus $y_{7}$ is adjacent to no vertex of cycle $C_{1}$, a contradiction.

Lemma 5.2: Let $G$ be a 3-connected graph of girth 5 such that all odd cycles of length greater than 5 have a chord. Let $C_{1}$ be a 5 -cycle in $G$. If $N^{\prime}\left(C_{1}\right)$ contains a cycle $C_{2}$, then $G \cong P_{10}$.

Proof: Denote $G\left[C_{1} \cup C_{2}\right]$ by $H$. By Lemma 5.1, $C_{2}$ is a 5 -cycle and $H$ is isomorphic to $P_{10}$. So let us adopt the notation that $C_{1}=x_{1} x_{2} x_{3} x_{4} x_{5} x_{1}, C_{2}=x_{6} x_{8} x_{10} x_{7} x_{9} x_{6}$ and each vertex $x_{i} \in V\left(C_{1}\right)$ is adjacent to the vertex $x_{i+5} \in V\left(C_{2}\right)$ where the subscripts are read modulo 5 .

Suppose $G \neq H$.

Claim 1: For $1 \leq i<j \leq 10, N_{i}^{\prime} \cap N_{j}^{\prime}=\emptyset$.

This follows by Lemma 2.4(i) and the girth hypothesis.

Claim 2: Let $B$ be a bridge of $H \cup N^{\prime}(H)$. Then $B$ cannot be an edge-bridge.

This follows by Lemma 2.4(iii) and Lemma 2.3. 
Claim 3: For any non-edge-bridge $B$, either $B$ is a monobridge $B_{i}$ or there exist $i, j \in[1,10]$ such that $B \cap N^{\prime}(H) \subseteq N_{i}^{\prime} \cup N_{j}^{\prime}$ where the corresponding $x_{i}$ and $x_{j}$ are two adjacent vertices in $H$. (That is to say, $B=B_{i, j}$ is a bibridge where $x_{i}$ and $x_{j}$ are adjacent.)

To prove Claim 3, first note that by Lemma 2.4(ii) and (iii) as well as Claim 2, every pair of non-adjacent vertices in $H$ are well-connected and hence form a non-cobridge pair. Any three vertices of $P_{10}$ must be such that at most two pair of them are adjacent. Therefore, no non-edge-bridge can have attachments in more than two $N_{i}^{\prime}$ 's, and if it has attachments in two $N_{i}^{\prime}$ s, say in $N_{i}^{\prime}$ and in $N_{j}^{\prime}$, then $x_{i}$ and $x_{j}$ are adjacent. This proves Claim 3.

Since $G$ is 3-connected, not all bridges of $H$ are monobridges. In fact, if a monobridge is joined only to $N_{i}^{\prime}$, then there must be a bibridge $B_{i, j}$ for some $j \neq i$, since $x_{i}$ is not a cutvertex in $G$. So let $B_{i, j}$ be a bibridge of $H$. Thus by Claim $3, x_{i} \sim x_{j}$.

Since $B_{i, j}$ is not an edge-bridge, there must exist an interior vertex $v$ of $B_{i, j}$. By Menger's theorem, there exist three internally disjoint paths from $v$ to three distinct vertices of $H$. Since $B_{i, j}$ is a bibridge, all attachments of $B_{i, j}$ in $H \cup N^{\prime}(H)$ are contained in $N_{i}^{\prime} \cup N_{j}^{\prime}$. Therefore, at least one of these three paths has to pass through a bridge different from $B_{i, j}$ which is either a $B_{i, k}$ bibridge or a $B_{j, k}$ bibridge, for some $k \neq i, j$. Without loss of generality, suppose one of the three paths passes through a $B_{j, k}$ bibridge. Hence, there must exist a path $P$ in $G \backslash V(H)$ joining $v$ to some vertex $w_{k}$ in $N_{k}^{\prime}, k \neq i, j$. Let $w_{i}$ be a neighbor of $v$ in $N_{i}^{\prime}$. $P+v w_{i}$ is then a path joining $w_{i}$ to $w_{k}$ in $G \backslash V(H)$. Now choose any shortest path $P_{i, k}$ joining a vertex $w_{i}$ of $N_{i}^{\prime}$ to a vertex $w_{k} \in N_{k}^{\prime}$. By Claim $3, x_{i}$ is adjacent to $x_{j}$ and $x_{j}$ is adjacent to $x_{k}$. Therefore, by the girth hypothesis, $x_{i}$ is not adjacent to $x_{k}$. (Note that $P_{i, k}$ may pass through monobridges of type $B_{j}$. See Figure 5.1.)

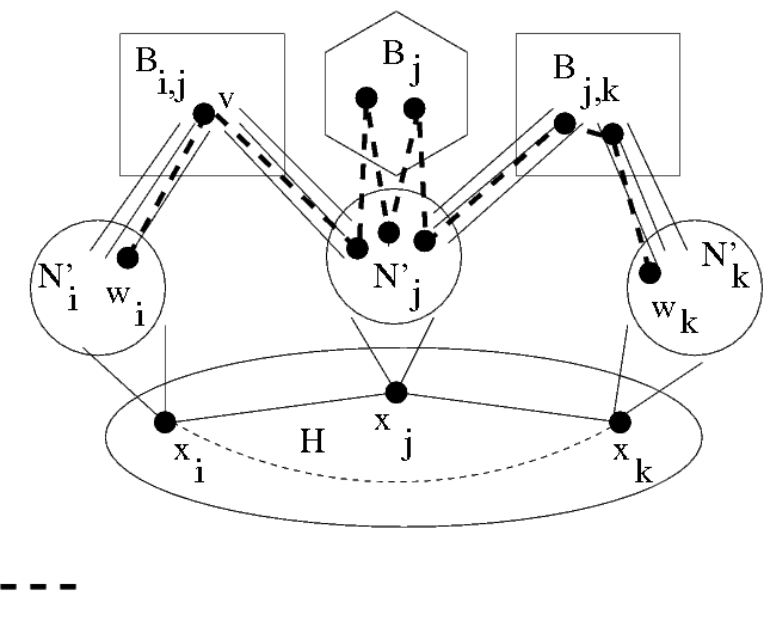

Figure 5.1

So by Lemma 2.5(i) and (iii), with $H \backslash x_{j}=P_{10} \backslash x_{j}$, there must exist an induced path $P_{1}$ of length 3 and an induced path $P_{2}$ of length 4 joining $x_{i}$ and $x_{k}$ in $H$, each 
avoiding vertex $x_{j}$. Let $Q_{3}=P_{i, k} \cup w_{i} x_{i} \cup w_{k} x_{k} \cup P_{1}$ and $Q_{4}=P_{i, k} \cup w_{i} x_{i} \cup w_{k} x_{k} \cup P_{2}$. Then $Q_{3}$ and $Q_{4}$ are chordless cycles since vertices in $G \backslash\left(H \cup N^{\prime}(H)\right)$ are not adjacent to vertices in $H, N^{\prime}(H)$ is independent, $N_{a}^{\prime} \cap N_{b}^{\prime}=\emptyset$, for $1 \leq a<b \leq 10$, and $P_{i, k}$ is chordless. But one of $Q_{3}$ and $Q_{4}$ is odd with length at least 9 , a contradiction.

Therefore no such vertex $v$ exists; i.e., $B_{i, j}=\emptyset$. Hence, since $G$ is 3 -connected, no $N_{i}^{\prime}$ 's exist either and it follows that $G=H \cong P_{10}$.

Lemma 5.3: Let $G$ be a 3-connected graph of girth five such that all odd cycles of length greater than 5 have a chord. Let $C_{1}$ be a 5 -cycle in $G$. Then if $N^{\prime}\left(C_{1}\right)$ contains an induced path $P$ of length at least $4, G \cong P_{10}$.

Proof: Let $C=x_{1} x_{2} x_{3} x_{4} x_{5} x_{1}$. By Lemma 5.2, we may assume $N^{\prime}(C)$ contains no cycle. By hypothesis, on the other hand, $N^{\prime}(C)$ contains an induced path $P$ of length at least 4. Let $H=G[C \cup P]$. Then it is easy to see that $H$ is isomorphic to $P_{10} \backslash u v$ for some pair of adjacent vertices $u$ and $v$.

Without loss of generality, we may assume that $H$ is labeled as in Figure 5.2.
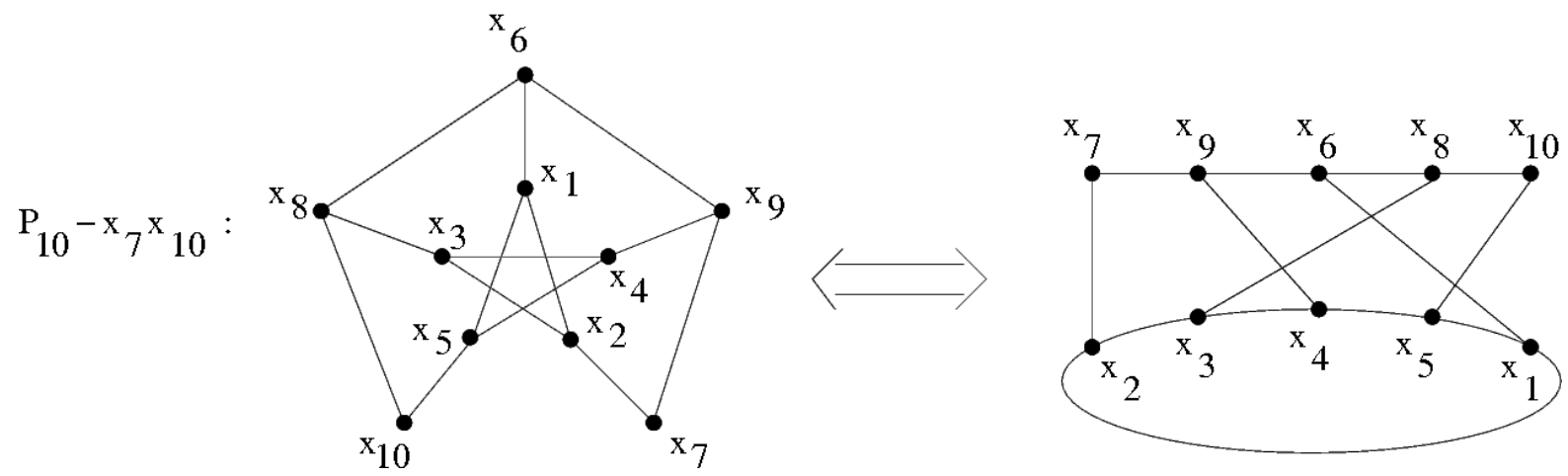

Figure 5.2

Claim 1: $N_{i}^{\prime} \cap N_{j}^{\prime}=\emptyset$, for $1 \leq i<j \leq 10$.

If the corresponding vertices $x_{i}$ and $x_{j}$ are at distance at most two in $H$, then $N_{i}^{\prime} \cap N_{j}^{\prime}=\emptyset$ by the girth hypothesis. There are five pairs of vertices at distance at least 3 , namely $\left\{x_{7}, x_{10}\right\},\left\{x_{2}, x_{10}\right\},\left\{x_{9}, x_{10}\right\},\left\{x_{5}, x_{7}\right\}$ and $\left\{x_{7}, x_{8}\right\}$. (See Figure 5.2.) Due to symmetry, we need only show that $N_{2}^{\prime} \cap N_{10}^{\prime}, N_{7}^{\prime} \cap N_{10}^{\prime}$ and $N_{9}^{\prime} \cap N_{10}^{\prime}$ are empty.

First, let us assume that $N_{2}^{\prime} \cap N_{10}^{\prime} \neq \emptyset$. Choose $w \in N_{2}^{\prime} \cap N_{10}^{\prime}$. Then $w$ is adjacent to both $x_{2}$ and $x_{10}$. But then $w x_{2} x_{7} x_{9} x_{6} x_{8} x_{10} w$ is a 7 -cycle, a contradiction. Similarly, if $N_{7}^{\prime} \cap N_{10}^{\prime} \neq \emptyset$ and $w \in N_{7}^{\prime} \cap N_{10}^{\prime}$, then $w x_{7} x_{2} x_{1} x_{6} x_{8} x_{10} w$ is a 7 -cycle and if $N_{9}^{\prime} \cap N_{10}^{\prime} \neq \emptyset$ and $w \in N_{9}^{\prime} \cap N_{10}^{\prime}$, then $w x_{9} x_{7} x_{2} x_{3} x_{8} x_{10} w$ is a 7 -cycle, a contradiction in each case. Thus Claim 1 is proved.

Claim 2: There exists no edge-bridge.

This follows from Lemma 2.5(ii) and Lemma 2.3. 
Claim 3: The only possible bridges are either monobridges or bibridges $B_{i, j}$ where $x_{i} \sim x_{j}$.

This is proved just as was Claim 3 of Lemma 5.2, except here we use Lemma 2.5(ii), instead of Lemma 2.4(ii) and (iii). So Claim 3 follows.

As noted in the proof of Lemma 5.2, not all bridges of $H$ are monobridges, so let $B_{i, j}$ be a bibridge. Hence by Claim 3 , vertices $x_{i}$ and $x_{j}$ are adjacent. By Claim $2, B_{i, j}$ is not an edge-bridge, so there must exist an interior vertex $v$ in $B_{i, j}$. Again arguing as in the proof of Lemma 5.2, there must exist a path $P$ in $G \backslash V(H)$ joining $v$ to a vertex $w_{k}$ in some $N_{k}^{\prime}, k \neq i, j$ and passing through a bibridge $B_{i, k}$ or a bibridge $B_{j, k}$. Say, without loss of generality, $P$ passes through a bibridge of type $B_{j, k}$. If $w_{i} \in N_{i}^{\prime}$, $P+v w_{i}$ is a path joining $w_{i}$ and $w_{k}$ in $G \backslash V(H)$. By Claim $3, x_{i} \sim x_{j}$ and $x_{j} \sim x_{k}$. But then by our girth hypothesis, $x_{i} \nsim x_{k}$. Therefore $\left\{x_{i}, x_{k}\right\}$ is a well-connected pair and by Lemma 2.5(iii) there exist induced paths of length 3 and 4 joining $x_{i}$ and $x_{k}$, both avoiding $x_{j}$.

Now arguing just as in the proof of Lemma 5.2, again we conclude that $B_{i, j}=\emptyset$ and the lemma follows.

Lemma 5.4: Let $G$ be a 3-connected graph of girth 5 such that all odd cycles of length greater than 5 have a chord. Let $C$ be a 5 -cycle in $G$. Then if $N^{\prime}(C)$ contains an induced path $P$ of length $3, G \cong P_{10}$.

Proof: Let $C=x_{1} x_{2} x_{3} x_{4} x_{5} x_{1}$ be a 5-cycle in $G$. By Lemma 5.3, $N^{\prime}(C)$ contains no induced path of length 4. Suppose, on the other hand, that $N^{\prime}(C)$ does contain an induced path $P$ of length 3 .

Suppose $G \approx P_{10}$.

Let $H=G[C \cup P]$. Then it is easy to see that $H=G[C \cup P]$ is isomorphic to $P_{10} \backslash v$ for some vertex $v$.

Let us suppose that $H$ is labeled as shown in Figure 5.3.

$\mathrm{H}:$
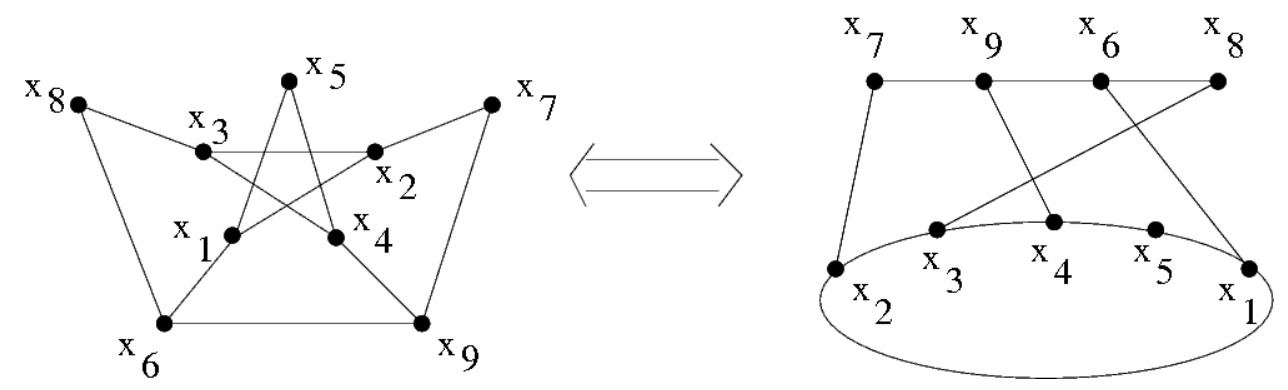

Figure 5.3

Claim 1: $N_{i}^{\prime} \cap N_{j}^{\prime}=\emptyset$, for $1 \leq i<j \leq 9$.

It is easy to check that every pair of vertices are at distance either 1 or 2 when one of the pair has degree 3. This implies that $N_{i}^{\prime} \cap N_{j}^{\prime}=\emptyset$ if one of $i$ and $j$ is not 5,7 or 8 . 
Suppose that, say, $N_{5}^{\prime} \cap N_{7}^{\prime} \neq \emptyset$ and we choose $w \in N_{5}^{\prime} \cap N_{7}^{\prime}$. Then $w$ is adjacent to both $x_{5}$ and $x_{7}$ and $H \cup w$ is a graph containing 5-cycle $C_{1}=x_{1} x_{5} x_{4} x_{9} x_{6} x_{1}$ together with a path $P_{C_{1}}=w x_{7} x_{2} x_{3} x_{8}$ in $N^{\prime}\left(C_{1}\right)$. But $P_{C_{1}}$ has length 4 and so by Lemma 5.3, $G \cong P_{10}$, a contradiction. So $N_{5}^{\prime} \cap N_{7}^{\prime}=\emptyset$.

By symmetry, the same argument may be used to show that if $G \approx P_{10}$, then $N_{5}^{\prime} \cap N_{8}^{\prime} \neq \emptyset$ and $N_{7}^{\prime} \cap N_{8}^{\prime} \neq \emptyset$.

Note that $H \cup w$ can viewed as follows. Add a vertex $w=x_{10}$ and join it to vertices $x_{5}, x_{7}$ and $x_{8}$ in Figure 5.3 and then delete either edge $x_{5} x_{10}, x_{7} x_{10}$ or $x_{8} x_{10}$. But these three graphs are each isomorphic to $P_{10} \backslash e$, where $e$ is any edge of $P_{10}$, since $P_{10}$ is edge-transitive.

Therefore Claim 1 is true.

Claim 2: There exists no edge-bridge.

This follows from the existence of a path of length 4 as pointed out in Lemmas $2.5(\mathrm{i})$ and 2.3 .

Claim 3: The only possible bridges are either monobridges or bibridges $B_{i, j}$ where $x_{i} \sim x_{j}$.

This is proved using Lemma 2.5(i) just as in the proof of Claim 3 of Lemma 5.3.

As noted in the proof of Lemma 5.2, not all bridges of $H$ are monobridges, so let $B_{i, j}$ be a bibridge. Hence by Claim 3 , vertices $x_{i}$ and $x_{j}$ are adjacent. By Claim $2, B_{i, j}$ is not an edge-bridge, so there must exist an interior vertex $v$ in $B_{i, j}$. Again arguing as in the proof of Lemma 5.2, there must exist a path $P$ in $G \backslash V(H)$ joining $v$ to a vertex $w_{k}$ in some $N_{k}^{\prime}, k \neq i, j$ and passing through a bibridge $B_{i, k}$ or a bibridge $B_{j, k}$. Say, without loss of generality, $P$ passes through a bibridge of type $B_{j, k}$. If $w_{i} \in N_{i}^{\prime}$, $P+v w_{i}$ is a path joining $w_{i}$ and $w_{k}$ in $G \backslash V(H)$. By Claim 3, $x_{i} \sim x_{j}$ and $x_{j} \sim x_{k}$. But then by our girth hypothesis, $x_{i} \nsim x_{k}$ and therefore $x_{i}$ and $x_{k}$ are well-connected and by Lemma 2.5(iii) there exist induced paths of length 3 and 4 joining $x_{i}$ and $x_{k}$, both avoiding vertex $x_{j}$.

Now arguing just as in the proof of Lemma 5.2, again we conclude that $B_{i, j}=\emptyset$ and the lemma follows. 


\section{Forbidden induced paths of length 2 .}

In this section, we will show the following. Suppose $G$ is 3 -connected, internally 4connected, has girth 5 and every odd cycle of length greater than 5 has a chord. Then if $C$ is a 5-cycle in $G$ such that $N^{\prime}(C)$ contains an induced path of length $2, G \cong P_{10}$.

So let us suppose that $C$ is a 5-cycle in $G$ and $N^{\prime}(C)$ does contain such an induced 2 -path $x_{3} x_{4} x_{5}$. Then by symmetry and the girth 5 hypothesis as well as 3 -connectivity, we may assume that $G$ contains the subgraph $J_{2}$ shown in Figure 3.2. (Note that $J_{2}$ must be induced by the girth 5 hypothesis.)

Our goal, then, is to show that if $G$ contains $J_{2}$ as a subgraph, then $G \cong P_{10}$. Let us point out again that we have not yet invoked the hypothesis of internal-4-connectivity.

Lemma 6.1: Suppose $G$ is 3-connected with girth 5 and every odd cycle of length greater than 5 has a chord. Suppose also that $G$ contains a subgraph isomorphic to $J_{2}$. Let the vertices of this $J_{2}$ be labeled as in Figure 3.2. Then if there exists an edge joining $N_{i}^{\prime}$ and $N_{j}^{\prime},\{i, j\}=\{1,3\},\{1,7\},\{3,5\}$, or $\{5,7\}$.

Proof: Suppose that there is an edge joining $u \in N_{1}^{\prime}$ to a vertex $v \in N_{i}^{\prime}$. Then $i \neq 1$, since $N_{1}^{\prime}$ is independent by the girth 5 hypothesis. For the same reason $i \neq 2,8$. Suppose $v \in N_{4}^{\prime}$. Then $u v x_{4} x_{5} x_{6} x_{2} x_{1} u$ is a 7-cycle, a contradiction of Lemma 2.3. Hence $v \notin N_{4}^{\prime}$ and, by symmetry, $v \notin N_{6}^{\prime}$. Similarly, $v \notin N_{5}^{\prime}$, since if it were, $u v x_{5} x_{4} x_{3} x_{2} x_{1} u$ would be a 7-cycle. So $\{1, j\}=\{1,3\}$ or $\{1,7\}$.

Now consider possible edges joining $N_{2}^{\prime}$ to $N_{i}^{\prime}, i \neq 2$. Since no edge joins $N_{1}^{\prime}$ and $N_{4}^{\prime}$, by symmetry, no edge joins $N_{2}^{\prime}$ and $N_{5}^{\prime}$. Suppose there is an edge joining $u \in N_{2}^{\prime}$ and $v \in N_{4}^{\prime}$. Then $u v x_{4} x_{8} x_{7} x_{6} x_{2} u$ is a 7 -cycle, a contradiction. Hence by the girth 5 hypothesis and symmetry, there is no edge joining $N_{2}^{\prime}$ to $N_{i}^{\prime}$, where $i \neq 2$. By symmetry, then, the proof of the Lemma is complete.

The preceding Lemma shows that the only possible "ears" attached to the subgraph $J_{2}$ belong to one of four classes, namely $\{i, j\}=\{1,3\},\{1,7\},\{3,5\}$ or $\{5,7\}$. We now proceed as follows:

(1) In Lemma 6.2, we show that $J_{2}$ cannot have ears from three (or more) of these four classes. That is to say, $G$ cannot contain configuration $J_{3}$ shown in Figure 6.1 as a subgraph.

(2) Using (1), we show that $J_{2}$ cannot possess ears from exactly two of the four classes. Here there are, up to isomorphism, two separate cases to treat. (See configuration $J_{4}$ in Figure 6.2 and configuration $J_{5}$ in Figure 6.3.) That $G$ cannot contain $J_{4}$ or $J_{5}$ as subgraphs is shown in Lemmas 6.3 and 6.4 respectively.

Note that in the proof of Lemma 6.4, we will use the assumption of internal-4connectivity for the first time.

(3) Using (2), we show in Lemma 6.5 that if $G$ contains $J_{1}$ shown in Figure 3.1 as a subgraph, then $G \cong P_{10}$. 
(4) And finally, using (3), we show by means of Lemma 6.6 that if $G$ contains the graph $J_{2}$ shown in Figure 3.2 as a subgraph, then $G \cong P_{10}$.

We have then shown that if $N^{\prime}(C)$ contains an induced path of length exactly 2 , then $G \cong P_{10}$.

Lemma 6.2: Let $G$ be a 3-connected graph of girth 5 such that all odd cycles of length greater than 5 have a chord. Then $G$ does not contain as a subgraph the graph $J_{3}$ shown in Figure 6.1.

Proof: Suppose, by way of contradiction, that $G$ does contain $J_{3}$ as a subgraph.

Cycle $C=x_{1} x_{2} \cdots x_{11} x_{1}$ has length 11 and hence contains a chord. Since the girth of $G$ is five and by Lemma 2.3 there are no 7 -cycles, the only possible chords of $C$ are of the form $x_{i} x_{i+4}$ or $x_{i} x_{i+7}$ (modulo 11). Therefore, $C$ can only have eleven possible chords: $x_{1} x_{5}, x_{2} x_{6}, x_{3} x_{7}, x_{4} x_{8}, x_{5} x_{9}, x_{6} x_{10}, x_{7} x_{11}, x_{1} x_{8}, x_{2} x_{9}, x_{3} x_{10}$ and $x_{4} x_{11}$. However, if $x_{1} x_{5} \in E(G)$, then $x_{1} x_{5} x_{6} x_{14} x_{9} x_{10} x_{11} x_{1}$ is a 7-cycle, if $x_{1} x_{8} \in E(G)$, then $x_{1} x_{8} x_{7} x_{6} x_{13} x_{3} x_{2} x_{1}$ is a 7-cycle, if $x_{2} x_{6} \in E(G)$, then $x_{2} x_{6} x_{13} x_{3} x_{2}$ is a 4-cycle, if $x_{2} x_{9} \in E(G)$, then $x_{2} x_{9} x_{14} x_{6} x_{5} x_{4} x_{3} x_{2}$ is a 7-cycle, if $x_{3} x_{7} \in E(G)$, then $x_{3} x_{7} x_{6} x_{13} x_{3}$ is a 4-cycle, if $x_{3} x_{10} \in E(G)$, then $x_{3} x_{10} x_{13} x_{3}$ is a 3-cycle, if $x_{4} x_{8} \in E(G)$, then $x_{4} x_{8} x_{9} x_{10} x_{11} x_{12} x_{3} x_{4}$ is a 7-cycle, if $x_{4} x_{11} \in E(G)$, then $x_{4} x_{11} x_{12} x_{3} x_{4}$ is a 4-cycle, if $x_{5} x_{9} \in E(G)$, then $x_{5} x_{9} x_{14} x_{6} x_{5}$ is a 4-cycle, if $x_{6} x_{10} \in E(G)$, then $x_{6} x_{10} x_{13} x_{6}$ is a 3-cycle, and if $x_{7} x_{11} \in E(G)$, then $x_{7} x_{11} x_{12} x_{3} x_{4} x_{5} x_{6} x_{7}$ is a 7 -cycle, so each of these possibilities also leads to a contradiction of either Lemma 2.3 or the girth hypothesis.

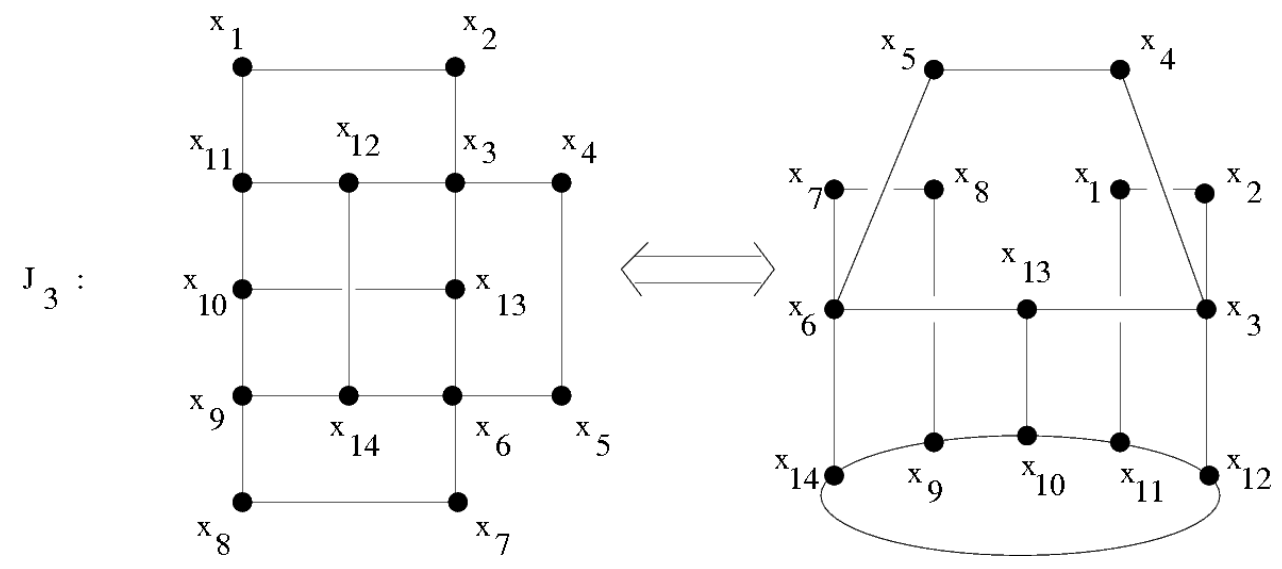

Figure 6.1

Lemma 6.3: Let $G$ be a 3-connected graph of girth 5 such that all odd cycles of length greater than 5 have a chord. Then $G$ does not contain a subgraph isomorphic to the graph $J_{4}$ shown in Figure 6.2. 


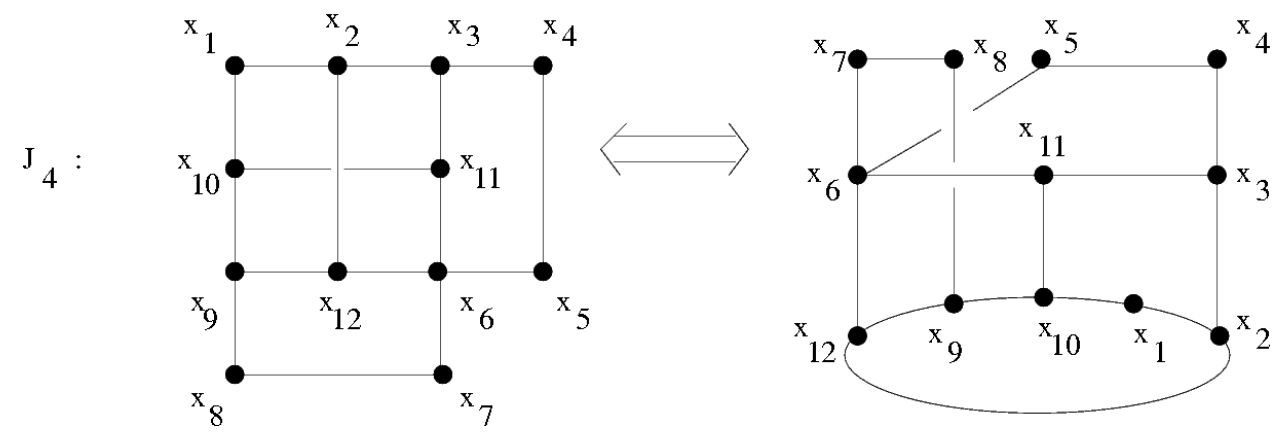

Figure 6.2

Proof: Claim 1: If $J_{4}$ is a subgraph of $G$, then it is an induced subgraph.

By the girth 5 assumption and symmetry, we need only check the pairs $\left\{x_{1}, x_{5}\right\}$, $\left\{x_{3}, x_{8}\right\}$ and $\left\{x_{4}, x_{8}\right\}$ for adjacency. But if $x_{1}$ and $x_{5}$ are adjacent, $x_{1} x_{5} x_{6} x_{7} x_{8} x_{9} x_{10} x_{1}$ is a 7-cycle, contradicting Lemma 2.3. Similarly, if $x_{3}$ and $x_{8}$ are adjacent, $x_{3} x_{8} x_{9} x_{12} x_{6} x_{5}$ $x_{4} x_{3}$ is a 7 -cycle, a contradicton, and if $x_{4}$ and $x_{8}$ are adjacent, $x_{4} x_{8} x_{9} x_{12} x_{6} x_{11} x_{3} x_{4}$ is a 7 -cycle, a contradiction. This proves Claim 1.

Claim 2: Let $x_{i}$ and $x_{j}$ be two nonadjacent vertices of $J_{4}$ and suppose that $x_{i} \in$ $\left\{x_{1}, x_{2}, x_{10}\right\}$. Then:

(i) there exists an induced even path of length at least 4 joining $x_{i}$ and $x_{j}$ and

(ii) there exists an induced odd path of length at least 3 joining $x_{i}$ and $x_{j}$ with two exceptions: $\{i, j\}=\{2,4\},\{8,10\}$.

(iii) If $x_{i} \nsim x_{j}$ and $\left\{x_{i}, x_{j}\right\} \neq\left\{x_{2}, x_{4}\right\},\left\{x_{8}, x_{10}\right\}$, then $\left\{x_{i}, x_{j}\right\}$ is a non-co-bridge pair.

Proof: This is easily checked.

Claim 3: For $1 \leq i<j \leq 12, N_{i}^{\prime} \cap N_{j}^{\prime}=\emptyset$.

Proof: Choose $v \in N_{i}^{\prime}, i=1, \ldots, 12$. By the girth hypothesis, $v$ is not incident to any $x_{j}$ with $d\left(x_{i}, x_{j}\right) \leq 2$. Since by Lemma 2.3 there is no 7 -cycle in $G, v$ is not adjacent to $x_{j}$ if $J_{4}$ contains a path of length 5 joining $x_{i}$ and $x_{j}$. By symmetry, we need only check $x_{i}=x_{1}, x_{2}, x_{3}, x_{4}, x_{5}, x_{6}, x_{11}$ versus $x_{j}$ when $j>i$. This is easily done.

Claim 4: There is no edge-bridge joining $N_{i}^{\prime}$ to $N_{j}^{\prime}$, for $1 \leq i<j \leq 12$.

Proof: Suppose, to the contrary, that $N^{\prime}\left(J_{4}\right)$ is dependent and hence there is an edge $u v$ joining the two vertices $u, v \in N^{\prime}\left(J_{4}\right)$ such that $u$ is adjacent to $x_{i}$ and $v$ is adjacent to $x_{j}$. Clearly, $x_{i}$ and $x_{j}$ are not adjacent by the girth hypothesis. By Claim 2, graph $J_{4}$ contains an induced even path $P_{i j}$ of length at least 4 joining $x_{i}$ and $x_{j}$. Then $Q=P_{i j} \cup\left\{u v, u x_{i}, v x_{j}\right\}$ is an odd cycle of length at least 7. But then $Q$ must contain a chord. However, by Claim 3, neither $u$ nor $v$ can be an endvertex of this chord. But then $P_{u v}$ is not induced, a contradiction. Therefore Claim 4 is true. 
Let $H=G\left[J_{4} \cup N^{\prime}\left(J_{4}\right)\right]$. We now consider the possible bridges of $H$. Let $\mathcal{B}_{i}$ denote the collection of all bridges $B$ of $H$ such that $B \cap N_{i}^{\prime} \neq \emptyset$; that is, $\mathcal{B}_{i}$ is the collection of bridges of $H$ having attachments in $N_{i}^{\prime}$.

Since $x_{1}$ has degree two in $J_{4}, N_{1}^{\prime} \neq \emptyset$. By Claims 3 and 4 , each vertex in $N_{1}^{\prime}$ has degree one in $H$ and hence $\mathcal{B}_{1} \neq \emptyset$.

Claim 5: Suppose $B \in \mathcal{B}_{1}$. Then one of the following three cases must hold:

(i) $B$ has all attachments contained in $N_{1}^{\prime}$; that is, $B=B_{1}$ is a monobridge of $H$;

(ii) $B$ has all attachments contained in $N_{1}^{\prime} \cup N_{2}^{\prime}$; that is, $B$ is a bibridge of type $B_{1,2}$; or

(iii) $B$ has all attachments contained in $N_{1}^{\prime} \cup N_{10}^{\prime}$; that is $B$ is a bibridge of type $B_{1,10}$.

Proof of Claim 5: By Claims 2, 3 and 4, $x_{1}$ and $x_{i}$ are non-co-bridge pairs, for $i \neq 1,2$ and 10. Therefore, Claim 5 is true.

Claim 6: Suppose $B \in \mathcal{B}_{2}$. Then one of the following must occur:

(i) $B$ has all attachments in $N_{2}^{\prime}$; that is, $B=B_{2}$ is a monobridge of $H$;

(ii) $B$ has all attachments in $N_{2}^{\prime} \cup N_{1}^{\prime}, N_{2}^{\prime} \cup N_{3}^{\prime}, N_{2}^{\prime} \cup N_{4}^{\prime}$ or in $N_{2}^{\prime} \cup N_{12}^{\prime}$; that is, $B$ is a bibridge of type $B_{2,1}$, type $B_{2,3}$, type $B_{2,4}$ or type $B_{2,12}$ respectively; or

(iii) $B$ has all attachments in $N_{2}^{\prime} \cup N_{3}^{\prime} \cup N_{4}^{\prime}$, and each of $N_{2}^{\prime}, N_{3}^{\prime}$ and $N_{4}^{\prime}$ contains at least one such attachment.

Proof of Claim 6: If $j \neq 4$ and $x_{2}$ and $x_{j}$ are not adjacent, then by Claim 2(iii), $\left\{x_{2}, x_{j}\right\}$ is a non-co-bridge pair. Therefore, all attachments of $B$ are contained in $N_{1}^{\prime} \cup$ $N_{2}^{\prime} \cup N_{3}^{\prime} \cup N_{4}^{\prime} \cup N_{12}^{\prime}$. Also by Claim 2(iii), $\left\{x_{1}, x_{3}\right\},\left\{x_{1}, x_{4}\right\},\left\{x_{1}, x_{12}\right\},\left\{x_{3}, x_{12}\right\}$ and $\left\{x_{4}, x_{12}\right\}$ are non-co-bridge pairs, so $B$ contains attachments in both $N_{2}^{\prime}$ and $N_{1}^{\prime}$ alone, in both $N_{2}^{\prime}$ and $N_{3}^{\prime}$ alone, in both $N_{2}^{\prime}$ and $N_{4}^{\prime}$ alone, in both $N_{2}^{\prime}$ and $N_{12}^{\prime}$ alone, or in each of $N_{2}^{\prime}, N_{3}^{\prime}$ and $N_{4}^{\prime}$. Thus Claim 6 is true.

We now proceed to complete the proof of the lemma.

Since $\left\{x_{2}, x_{10}\right\}$ is a 2 -vertex cut in $J_{4}$ and $G$ is 3 -connected, there must exist paths from $x_{1}$ which pass through some vertices in $N_{1}^{\prime}$, then (by Claim 5) through some bridge of type $B_{1,2}$ ( or type $B_{1,10}$ ), and then, perhaps, through some other bridges in $\mathcal{B}_{2}$ (or $\left.\mathcal{B}_{10}\right)$ to vertices in $N_{j}^{\prime}$, for each $j \in S=\{3,4,5,6,7,8,9,11,12\}$. Let $Q$ be a shortest of these paths joining $x_{1}$ to some $x_{k} \in S$. By the minimality of the length of $Q, Q$ contains only one vertex in $N_{1}^{\prime}$ and hence $Q$ does not pass through both a bibridge of type $B_{1,2}$ and one of type $B_{1,10}$. By symmetry, we may assume $Q$ passes through a bridge of type $B_{1,2}$. Then by Claim $6, Q$ must pass some bridge of type $B_{2,3}$ to reach $x_{3}$, one of type $B_{2,4}$ to reach $x_{4}$, one of type $B_{2,12}$ to reach $x_{12}$, or some bridge $B$ having all attachments in $N_{2}^{\prime} \cup N_{3}^{\prime} \cup N_{4}^{\prime}$ to reach either $x_{3}$ or $x_{4}$.

First we suppose that $Q$ passes through a bridge of type $B_{2,3}$ to reach $x_{3}$. Since $x_{1}$ and $x_{3}$ are connected by the odd path $P_{1}=x_{1} x_{10} x_{11} x_{3}$ in $J_{5}$ and the even path $P_{2}=x_{1} x_{10} x_{9} x_{8} x_{7} x_{6} x_{5} x_{4} x_{3}$, both of which avoid $x_{2}$, both $Q \cup P_{1}$ and $Q \cup P_{2}$ are 
chordless cycles of opposite parity and each has length greater than 5 . So once more we get a chordless odd cycle of length greater than 5, a contradiction. (Note that since both $P_{1}$ and $P_{2}$ avoid $x_{2}$, this prevents possible chords joining $x_{2}$ and a vertex in $N_{2}^{\prime}$.)

Now we suppose that $Q$ passes through a bridge of type $B_{2,12}$ to reach $x_{12}$. Using the same argument with the odd path $P_{1}=x_{1} x_{10} x_{9} x_{12}$ and the even path $P_{2}=x_{1} x_{10} x_{11} x_{6} x_{12}$, both of which avoid $x_{2}$, we obtain a chordless odd cycle of length greater than 5 , a contradiction.

For the case of a bridge of type $B_{2,4}$, we use the same argument with the induced odd path $P_{1}=x_{1} x_{10} x_{9} x_{8} x_{7} x_{6} x_{5} x_{4}$ and the induced even path $P_{2}=x_{1} x_{10} x_{9} x_{12} x_{6} x_{5} x_{4}$, both of which avoid $x_{2}$, to obtain a chordless odd cycle of length greater than 5 , a contradiction.

The last case occurs when $Q$ passes through a bridge $B$ having all attachments in $N_{2}^{\prime} \cup N_{3}^{\prime} \cup N_{4}^{\prime}$ and, moreover, has attachments in each of the three. Since $Q$ is a shortest path, $Q$ contains only one vertex $w$ in $N_{3}^{\prime} \cup N_{4}^{\prime}$. If $w \in N_{3}^{\prime}$, then $x_{3} \in V(Q)$ and $Q \cap N_{4}^{\prime}=\emptyset$ and if $w \in N_{4}^{\prime}, x_{4} \in V(Q)$ and $Q \cap N_{3}^{\prime}=\emptyset$.

Suppose $x_{3} \in V(Q)$. Then $Q\left[x_{1}, x_{3}\right] \cup x_{1} x_{10} x_{11} x_{3}$ and $Q\left[x_{1}, x_{3}\right] \cup x_{1} x_{10} x_{9} x_{8} x_{7} x_{6} x_{5}$ $x_{4} x_{3}$ are of opposite parity. Moreover, both avoid vertex $x_{2}$ and hence are chordless. So $x_{1}$ and $x_{3}$ lie on a chordless odd cycle of length greater than 5. Suppose, then, that $x_{4} \in V(Q)$. Then $Q\left[x_{1}, x_{4}\right] \cup x_{1} x_{10} x_{9} x_{12} x_{6} x_{5} x_{4}$ and $Q\left[x_{1}, x_{4}\right] \cup x_{1} x_{10} x_{9} x_{8} x_{7} x_{6} x_{5} x_{4}$ are of opposite parity, both fail to contain both $x_{2}$ and $x_{3}$ and hence are chordless. Hence $x_{1}$ and $x_{4}$ must lie on a chordless odd cycle of length greater than 5 . This completes the proof of Lemma 6.3.

In Lemma 6.4, we assume internal-4-connectivity for the first time in eliminating the subgraph $J_{5}$. In fact, we point out that the counterexamples presented at the end of Section 3 are constructed by beginning with a subgraph isomorphic to $J_{5}$.

Lemma 6.4: Let $G$ be a 3-connected internally-4-connected graph of girth 5 such that all odd cycles of length greater than 5 have a chord. Then $G$ contains no subgraph isomorphic to graph $J_{5}$ shown in Figure 6.3.

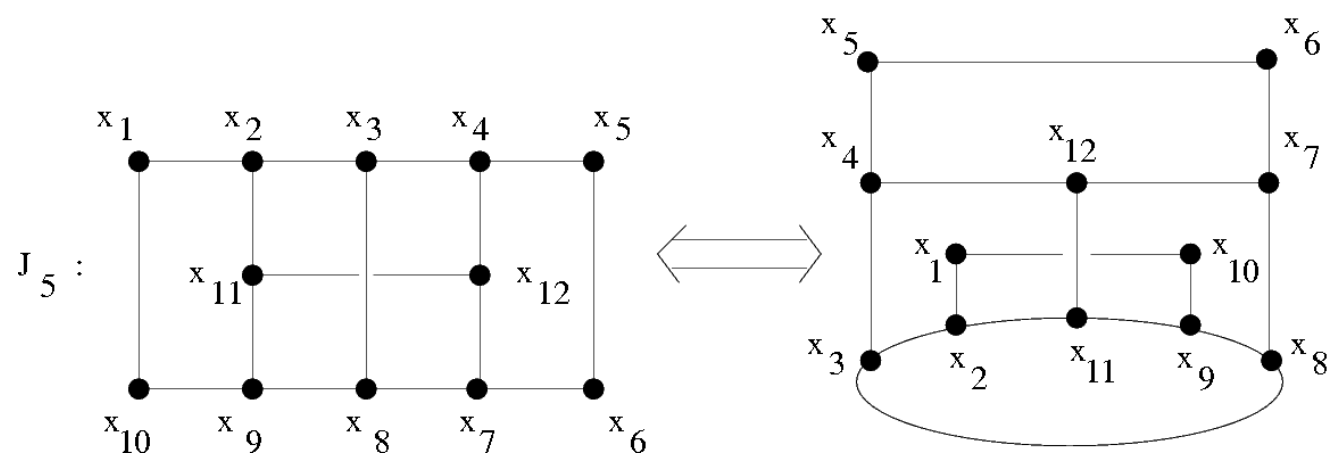

Figure 6.3

Proof: Suppose $G$ does contain $J_{5}$ as a subgraph. 
Claim 1: $J_{5}$ is an induced subgraph.

Proof of Claim 1: If $x_{1}$ and $x_{5}$ are adjacent, then $x_{1} x_{5} x_{6} x_{7} x_{8} x_{9} x_{10} x_{1}$ is a 7-cycle, if $x_{1}$ and $x_{6}$ are adjacent, then $x_{1} x_{6} x_{7} x_{8} x_{9} x_{11} x_{2} x_{1}$ is a 7-cycle and if $x_{1}$ and $x_{7}$ are adjacent, then $x_{1} x_{2} x_{3} x_{4} x_{5} x_{6} x_{7} x_{1}$ is a 7 -cycle. But by Lemma 2.3 there are no 7 -cycles.

Any other possible chord in $J_{5}$ would violate the girth 5 hypothesis.

Claim 2: For $1 \leq i<j \leq 12, N_{i}^{\prime} \cap N_{j}^{\prime}=\emptyset$.

By the girth 5 hypothesis, $N_{1}^{\prime} \cap N_{2}^{\prime}=N_{1}^{\prime} \cap N_{3}^{\prime}=N_{1}^{\prime} \cap N_{9}^{\prime}=N_{1}^{\prime} \cap N_{10}^{\prime}=N_{1}^{\prime} \cap N_{11}^{\prime}=$ $N_{2}^{\prime} \cap N_{3}^{\prime}=N_{2}^{\prime} \cap N_{4}^{\prime}=N_{2}^{\prime} \cap N_{8}^{\prime}=N_{2}^{\prime} \cap N_{9}^{\prime}=N_{2}^{\prime} \cap N_{10}^{\prime}=N_{2}^{\prime} \cap N_{11}^{\prime}=N_{2}^{\prime} \cap N_{12}^{\prime}=N_{3}^{\prime} \cap N_{4}^{\prime}=$ $N_{3}^{\prime} \cap N_{5}^{\prime}=N_{3}^{\prime} \cap N_{7}^{\prime}=N_{3}^{\prime} \cap N_{8}^{\prime}=N_{3}^{\prime} \cap N_{9}^{\prime}=N_{3}^{\prime} \cap N_{11}^{\prime}=N_{3}^{\prime} \cap N_{12}^{\prime}=N_{11}^{\prime} \cap N_{12}^{\prime}=\emptyset$.

The following pairs of vertices $\left\{x_{i}, x_{j}\right\}$ are joined by the indicated paths of length 5. Hence $N_{i}^{\prime} \cap N_{j}^{\prime}=\emptyset$ for these pairs since $G$ has no 7-cycle.

$\left\{x_{1}, x_{4}\right\}: x_{1} x_{10} x_{9} x_{8} x_{3} x_{4} ;\left\{x_{1}, x_{5}\right\}: x_{1} x_{2} x_{11} x_{12} x_{4} x_{5} ;\left\{x_{1}, x_{6}\right\}: x_{1} x_{2} x_{3} x_{4} x_{5} x_{6} ;$ $\left\{x_{1}, x_{7}\right\}: x_{1} x_{2} x_{3} x_{4} x_{12} x_{7} ;\left\{x_{1}, x_{8}\right\}: x_{1} x_{2} x_{11} x_{12} x_{7} x_{8} ;\left\{x_{1}, x_{12}\right\}: x_{1} x_{2} x_{3} x_{8} x_{7} x_{12} ;$ $\left\{x_{2}, x_{5}\right\}: x_{2} x_{3} x_{8} x_{7} x_{6} x_{5} ;\left\{x_{2}, x_{6}\right\}: x_{2} x_{11} x_{9} x_{8} x_{7} x_{6} ;\left\{x_{2}, x_{7}\right\}: x_{2} x_{1} x_{10} x_{9} x_{8} x_{7} ;\left\{x_{3}, x_{6}\right\}:$ $x_{3} x_{2} x_{11} x_{12} x_{7} x_{6}$.

All remaining pairs are symmetric to one of those listed above and hence Claim 2 follows.

Claim 3: If $\{i, j\} \notin\{\{1,11\},\{5,12\},\{6,12\},\{10,11\},\{2,9\},\{4,7\}\}$, then there is no edge joining $N_{i}^{\prime}$ and $N_{j}^{\prime}$.

If $\{i, j\}=\{1,2\},\{1,10\},\{2,3\},\{2,11\},\{3,4\},\{3,8\},\{11,12\}$, then no edge joins $N_{i}^{\prime}$ and $N_{j}^{\prime}$ by the girth five hypothesis.

For the following pairs $\left\{x_{i}, x_{j}\right\}$, we list either an induced 4-path, an induced 6-path or an induced 8-path joining $x_{i}$ and $x_{j}$. If there is an edge joining $N_{i}^{\prime}$ and $N_{j}^{\prime}$ in these cases, we get either a 7-cycle, which contradicts Lemma 2.3, a chordless 9-cycle or a chordless 11-cycle which contradicts the girth hypothesis of the present Lemma.

$\left\{x_{1}, x_{3}\right\}: x_{1} x_{10} x_{9} x_{8} x_{3} ;\left\{x_{1}, x_{4}\right\}: x_{1} x_{2} x_{11} x_{12} x_{4} ;\left\{x_{1}, x_{5}\right\}: x_{1} x_{2} x_{3} x_{4} x_{5} ;\left\{x_{1}, x_{6}\right\}:$ $x_{1} x_{2} x_{11} x_{9} x_{8} x_{7} x_{6} ;\left\{x_{1}, x_{7}\right\}: x_{1} x_{2} x_{3} x_{8} x_{7} ;\left\{x_{1}, x_{8}\right\}: x_{1} x_{2} x_{11} x_{9} x_{8} ;\left\{x_{1}, x_{9}\right\}: x_{1} x_{2} x_{3} x_{8} x_{9}$; $\left\{x_{1}, x_{12}\right\}: x_{1} x_{2} x_{3} x_{4} x_{12} ;\left\{x_{2}, x_{4}\right\}: x_{2} x_{1} x_{10} x_{9} x_{8} x_{7} x_{6} x_{5} x_{4} ;\left\{x_{2}, x_{5}\right\}: x_{2} x_{11} x_{12} x_{4} x_{5} ;$ $\left\{x_{2}, x_{6}\right\}: x_{2} x_{11} x_{12} x_{7} x_{6} ;\left\{x_{2}, x_{7}\right\}: x_{2} x_{3} x_{4} x_{12} x_{7} ;\left\{x_{2}, x_{8}\right\}: x_{2} x_{1} x_{10} x_{9} x_{8} ;\left\{x_{2} x_{10}\right\}:$ $x_{2} x_{3} x_{8} x_{9} x_{10} ;\left\{x_{2}, x_{12}\right\}: x_{2} x_{3} x_{8} x_{7} x_{12} ;\left\{x_{3}, x_{5}\right\}: x_{3} x_{8} x_{7} x_{6} x_{5} ;\left\{x_{3}, x_{6}\right\}: x_{3} x_{4} x_{12} x_{7} x_{6}$; $\left\{x_{3}, x_{7}\right\}: x_{3} x_{2} x_{11} x_{12} x_{7} ;\left\{x_{3}, x_{11}\right\}: x_{3} x_{8} x_{7} x_{12} x_{11}$.

All other pairs are symmetric to one of those listed above. This proves Claim 3.

Let $\mathcal{S}_{1}=\{1,2,9,10\}$ and $\mathcal{S}_{2}=\{4,5,6,7,12\}$; that is, the vertex partition in $J_{5}$ induced by the 3 -cut $\left\{x_{3}, x_{8}, x_{11}\right\}$.

Claim 4: The following pairs of vertices are well-connected in $J_{3}$ and, except for $\left\{x_{1}, x_{10}\right\}$ and $\left\{x_{10}, x_{11}\right\}$, each is a non-co-bridge pair. 
(i) all $\left\{x_{i}, x_{j}\right\}$ where $i \in \mathcal{S}_{1}$ and $j \in \mathcal{S}_{2}$, and

(ii) $\left\{x_{3}, x_{6}\right\},\left\{x_{3}, x_{7}\right\},\left\{x_{3}, x_{9}\right\},\left\{x_{3}, x_{10}\right\},\left\{x_{3}, x_{11}\right\},\left\{x_{3}, x_{12}\right\},\left\{x_{1}, x_{8}\right\},\left\{x_{2}, x_{8}\right\},\left\{x_{4}, x_{8}\right\}$, $\left\{x_{5}, x_{8}\right\},\left\{x_{8}, x_{11}\right\},\left\{x_{8}, x_{12}\right\},\left\{x_{1}, x_{11}\right\},\left\{x_{4}, x_{11}\right\},\left\{x_{5}, x_{11}\right\},\left\{x_{6}, x_{11}\right\},\left\{x_{7}, x_{11}\right\}$, $\left\{x_{10}, x_{11}\right\}$.

The reader may easily check that each of the pairs in (1) and (2) are well-connected.

By Claim 3, except for $\left\{x_{1}, x_{10}\right\}$ and $\left\{x_{10}, x_{11}\right\}$, there do not exist edges between $N_{i}^{\prime}$ and $N_{j}^{\prime}$, for the pairs $\left\{x_{i}, x_{j}\right\}$ listed in (i) and (ii). Hence the Claim follows.

For each $i=1, \ldots, 12$, define

$\mathcal{B}_{i}=\left\{B \mid B\right.$ is a non - edge bridge of $J_{3} \cup N^{\prime}\left(J_{3}\right)$ such that $B$ has attachments in $\left.N_{i}^{\prime}\right\}$.

Since $\left\{x_{3}, x_{8}, x_{11}\right\}$ is a 3 -vertex cut in $J_{3}$, and since $G$ is internally 4 -connected, there must exist paths from each vertex in $\mathcal{S}_{1}$ to each vertex in $\mathcal{S}_{2}$ which do not pass through any of $x_{3}, x_{8}$ or $x_{11}$. By Claim 3, there are no edges joining $N_{i}^{\prime}$ to $N_{j}^{\prime}$, for all $i, j, i \in \mathcal{S}_{1}, j \in \mathcal{S}_{2}$. By Claim 4(i), for each $i \in \mathcal{S}_{1}$ and each $j \in \mathcal{S}_{2},\left\{x_{i}, x_{j}\right\}$ is a non-co-bridge pair and hence any such path joining $\mathcal{S}_{1}$ and $\mathcal{S}_{2}$ must pass through some vertices in $N_{3}^{\prime} \cup N_{8}^{\prime} \cup N_{11}^{\prime}$ and through some non-edge bridges in $\mathcal{B}_{3} \cup \mathcal{B}_{8} \cup \mathcal{B}_{11}$.

Now let $Q$ be a shortest such path from $\left\{x_{i} \mid i \in \mathcal{S}_{1}\right\}$ to $\left\{x_{j} \mid j \in \mathcal{S}_{2}\right\}$, which does not pass through $x_{3}, x_{8}$ or $x_{11}$. By minimality of the length of $Q, Q$ intersects only one $N_{i}^{\prime}, i \in \mathcal{S}_{1}$, and that in only one vertex and $Q$ intersects only one $N_{j}^{\prime}, j \in \mathcal{S}_{2}$, and that in only one vertex. Since $\left\{x_{3}, x_{11}\right\}$ and $\left\{x_{8}, x_{11}\right\}$ are non-co-bridge pairs, $\mathcal{B}_{3} \cap \mathcal{B}_{11}=\mathcal{B}_{8} \cap \mathcal{B}_{11}=\emptyset$. But $Q$ must meet $N_{3}^{\prime} \cup N_{8}^{\prime} \cup N_{11}^{\prime}$. Therefore, $Q$ can only meet $N_{11}^{\prime}$ or $N_{3}^{\prime} \cup N_{8}^{\prime}$, but not both. So we have two cases.

Case 1. Suppose $Q$ leaves $\mathcal{S}_{1}$ via one of $x_{1}$ or $x_{10}$, say without loss of generality by symmetry, via vertex $x_{1}$. Since $\{1,8\}$ is a non-co-bridge pair, $Q$ must use either a bibridge of type $B_{1,3}$ or of type $B_{1,11}$, but not both, by the minimality of the length of $Q$.

Case 1.1. Suppose $Q$ uses a bibridge of type $B_{1,3}$. If $Q$ then traverses a bridge of type $B_{3,8}$, the induced paths $x_{1} x_{2} x_{11} x_{9} x_{8}$ and $x_{1} x_{10} x_{9} x_{8}$ (both of which avoid vertex $x_{3}$ ) guarantee the existence of a chordless odd cycle of length greater than 5 , a contradiction.

On the other hand, if $Q$ traverses a bridge of type $B_{3,4}$ after traversing $B_{1,3}$, the induced paths $x_{1} x_{10} x_{9} x_{8} x_{7} x_{12} x_{4}$ and $x_{1} x_{10} x_{9} x_{11} x_{12} x_{4}$ (both of which avoid vertex $x_{3}$ ) guarantee a chordless odd cycle of length greater than 5, again a contradiction.

This completes the proof in Case 1.1, since the remaining possible pairs of type $\left\{x_{3}, x_{j}\right\}$, namely those for which $j=5,6,7,12$ are all non-co-bridge pairs.

Case 1.2. Suppose, on the other hand, that $Q$ uses a bibridge of type $B_{1,11}$. Then, since $\left\{x_{4}, x_{11}\right\},\left\{x_{5}, x_{11}\right\},\left\{x_{6}, x_{11}\right\}$ and $\left\{x_{7}, x_{11}\right\}$ are non-co-bridge pairs, $Q$ must next traverse a bibridge of type $B_{11,12}$. The induced paths $x_{1} x_{2} x_{3} x_{4} x_{12}$ and $x_{1} x_{10} x_{9} x_{8} x_{7} x_{12}$ 
(both of which avoid vertex $x_{11}$ ) then guarantee a chordless odd cycle of length greater than 5 , a contradiction.

Case 2. Suppose now that $Q$ leaves $\mathcal{S}_{1}$ via one of $x_{2}$ or $x_{9}$, say without loss of generality by symmetry, via $x_{2}$. Since $\left\{x_{2}, x_{8}\right\}$ is a non-co-bridge pair, $Q$ must traverse a bibridge of type $B_{2,3}$ or one of type $B_{2,11}$ (but not both).

Case 2.1. Suppose first that $Q$ uses a bibridge of type $B_{2,3}$.

Suppose $Q$ next traverses a bridge of type $B_{3, j}$. Since $\left\{x_{3}, x_{j}\right\}$, for $j=6,7,12$ is a non-co-bridge pair, there are just three cases to treat.

If $j=4$, induced paths $x_{2} x_{11} x_{12} x_{4}$ and $x_{2} x_{1} x_{10} x_{9} x_{8} x_{7} x_{6} x_{5} x_{4}$ (both of which avoid vertex $x_{3}$ ) guarantee a chordless odd cycle of length greater than 5 . If $j=5$, induced paths $x_{2} x_{11} x_{12} x_{4} x_{5}$ and $x_{2} x_{11} x_{12} x_{7} x_{6} x_{5}$ (both of which avoid $x_{3}$ ) imply a chordless odd cycle of length greater than 5. Finally, if $j=8$, induced paths $x_{2} x_{11} x_{9} x_{8}$ and $x_{2} x_{1} x_{10} x_{9} x_{8}$ (both of which avoid $x_{3}$ ) guarantee a chordless odd cycle of length greater than 5 . In all three instances we obtain a contradiction.

Case 2.2. Suppose $Q$ next traverses a bibridge of type $B_{2,11}$.

Since $\left\{x_{4}, x_{11}\right\},\left\{x_{5}, x_{11}\right\},\left\{x_{6}, x_{11}\right\}$ and $\left\{x_{7}, x_{11}\right\}$ are all non-co-bridge pairs, $Q$ must next traverse a bibridge of type $B_{11,12}$. But in this instance induced paths $x_{2} x_{3} x_{4} x_{12}$ and $x_{2} x_{3} x_{8} x_{7} x_{12}$ (both of which avoid $x_{11}$ ) imply the existence of a chordless odd cycle of length greater than 5 , a contradiction.

Lemma 6.5: Let $G$ be a 3 -connected internally-4-connected graph of girth 5 such that all odd cycles of length greater than 5 have a chord. Then if $G$ contains a subgraph isomorphic to graph $J_{1}$ shown in Figure $3.1, G \cong P_{10}$.

Proof: Suppose $G$ does contain a subgraph isomorphic to $J_{1}$. Let us assume the vertex labelling shown in Figure 3.1.

Suppose $G \neq P_{10}$.

Claim 1: The subgraph $J_{1}$ must be induced.

It is easy to check that adding any edge different from $x_{1} x_{7}$ and $x_{4} x_{10}$ results in the formation of a cycle of size less than five, contradicting the girth hypothesis.

So then let us assume $x_{1}$ is adjacent to $x_{7}$. Then if $C=x_{2} x_{3} x_{8} x_{9} x_{11} x_{2}, N^{\prime}(C)$ contains the induced path $x_{10} x_{1} x_{7} x_{12} x_{4}$ of length 4, contradicting Lemma 5.3. By symmetry, if we add the edge $x_{4} x_{10}$, a similar contradiction is reached. This proves Claim 1.

Claim 2: For $1 \leq i<j \leq 12, N_{i}^{\prime} \cap N_{j}^{\prime}=\emptyset$.

It is routine to check that any possible non-empty intersection of two different $N_{i}^{\prime}$ 's produces either a cycle of length less than 5 , thus contradicting the girth hypothesis, or else a 7 -cycle, thus contradicting Lemma 2.3. This proves Claim 2. 
Claim 3: For all pairs $\{i, j\}, 1 \leq i<j \leq 12$, except $\{1,11\},\{10,11\}$, and $\{2,9\}$, there is no edge joining $N_{i}^{\prime}$ and $N_{j}^{\prime}$.

For all pairs $\{i, j\}$ in the pair set under consideration, except $\{2,4\},\{4,7\}$, and $\{2,9\}$, if there is an edge joining $N_{i}^{\prime}$ and $N_{j}^{\prime}$, there results either a cycle of length less than 5 (contradicting the girth hypothesis) or a 7-cycle (contradicting Lemma 2.3). On the other hand, if there is an edge joining $N_{2}^{\prime}$ and $N_{4}^{\prime}$, this results in a subgraph isomorphic to $J_{4}$ and hence by Lemma $6.3, G \cong P_{10}$, a contradiction, while if there is an edge joining $N_{4}^{\prime}$ and $N_{7}^{\prime}$, we get a subgraph isomorphic to $J_{5}$ contradicting Lemma 6.4. This proves Claim 3.

Let $\mathcal{S}_{1}=\{1,2,9,10\}$ and $\mathcal{S}_{2}=\{4,7,12\}$; i.e., the partition in $J_{1}$ induced by the 3-cut $\left\{x_{3}, x_{8}, x_{11}\right\}$.

Claim 4: The following pairs of vertices are well-connected in $J_{5}$ and hence, except for $\left\{x_{1}, x_{11}\right\}$ and $\left\{x_{10}, x_{11}\right\}$, each is a non-co-bridge-pair.

(i) all $\{i, j\}$ where $i \in \mathcal{S}_{1}$ and $j \in \mathcal{S}_{2}$, and

(ii) $\{1,8\},\{1,11\},\{2,8\},\{3,7\},\{3,9\},\{3,10\},\{3,11\},\{3,12\},\{4,8\},\{4,11\},\{7,11\}$, $\{8,11\},\{8,12\},\{10,11\}$.

Again, this is easily checked.

For each $i=1,2,3,4,7,8,9,10,11,12$, define $\mathcal{B}_{i}$ as in the proof of Claim 4 of Lemma 6.4 .

Since $\left\{x_{3}, x_{8}, x_{11}\right\}$ is a 3 -vertex cut in $J_{5}$, and since $G$ is internally 4 -connected, there must exist paths from each vertex in $\mathcal{S}_{1}$ to each vertex in $\mathcal{S}_{2}$ which do not pass through any of $x_{3}, x_{8}$ or $x_{11}$. By Claim 3, there are no edges joining $N_{i}^{\prime}$ to $N_{j}^{\prime}$, for all $i, j, i \in \mathcal{S}_{1}, j \in \mathcal{S}_{2}$. By Claim 4(i), for each $i \in \mathcal{S}_{1}$ and each $j \in \mathcal{S}_{2},\left\{x_{i}, x_{j}\right\}$ is a non-co-bridge pair and hence any such path joining $\mathcal{S}_{1}$ and $\mathcal{S}_{2}$ must pass through some vertices in $N_{3}^{\prime} \cup N_{8}^{\prime} \cup N_{11}^{\prime}$ and through some non-edge bridges in $\mathcal{B}_{3} \cup \mathcal{B}_{8} \cup \mathcal{B}_{11}$.

Now let $Q$ be a shortest such path from $\left\{x_{i} \mid i \in \mathcal{S}_{1}\right\}$ to $\left\{x_{j} \mid j \in \mathcal{S}_{2}\right\}$, which does not pass through $x_{3}, x_{8}$ or $x_{11}$. By minimality of the length of $Q, Q$ intersects only one $N_{i}^{\prime}, i \in \mathcal{S}_{1}$, and that in only one vertex and $Q$ intersects only one $N_{j}^{\prime}, j \in \mathcal{S}_{2}$, and that in only one vertex. Since $\left\{x_{3}, x_{11}\right\}$ and $\left\{x_{8}, x_{11}\right\}$ are non-co-bridge pairs, $\mathcal{B}_{3} \cap \mathcal{B}_{11}=\mathcal{B}_{8} \cap \mathcal{B}_{11}=\emptyset$. But $Q$ must meet $N_{3}^{\prime} \cup N_{8}^{\prime} \cup N_{11}^{\prime}$. Therefore, $Q$ can only meet $N_{11}^{\prime}$ or $N_{3}^{\prime} \cup N_{8}^{\prime}$, but not both. So we have two cases.

Case 1: Suppose $Q$ leaves $\mathcal{S}_{1}$ via one of $x_{2}$ or $x_{9}$, say without loss of generality by symmetry, via $x_{2}$. Since $\left\{x_{2}, x_{8}\right\}$ is a non-co-bridge pair, $Q$ must use a bibridge of type $B_{2,3}$ or $B_{2,11}$ (but not both).

Case 1.1. Suppose $Q$ traverses a bibridge of type $B_{2,3}$. Then $Q$ must next traverse a bibridge of type $B_{3, j}$, where $j \neq 7,11,12$, since $\left\{x_{3}, x_{7}\right\},\left\{x_{3}, x_{11}\right\}$ and $\left\{x_{3} x_{12}\right\}$ are nonco-bridge pairs. There are then only two possibilities. If $B_{3, j}=B_{3,4}$, the induced paths 
$x_{2} x_{11} x_{12} x_{4}$ and $x_{2} x_{11} x_{9} x_{8} x_{7} x_{12} x_{4}$ (both of which avoid vertex $x_{3}$ ) imply the existence of a chordless odd cycle of length greater than 5 , a contradiction.

If on the other hand, $B_{3, j}=B_{3,8}$, the induced paths $x_{2} x_{11} x_{9} x_{8}$ and $x_{2} x_{1} x_{10} x_{9} x_{8}$ (both of which avoid $x_{3}$ ) imply the existence of a chordless odd cycle of length greater than 5, again a contradiction.

Case 1.2. Suppose then that $Q$ traverses a bibridge of type $B_{2,11}$. If then $Q$ traverses a bibridge of type $B_{11, j}$, since $\left\{x_{11}, x_{j}\right\}$ is a non-co-bridge pair for $j=3,4,7,8$, the only possibility is that $Q$ traverses a bibridge of type $B_{11,12}$. However, in this case the induced paths $x_{2} x_{3} x_{4} x_{12}$ and $x_{2} x_{3} x_{8} x_{7} x_{12}$ (both of which avoid $x_{11}$ ) imply the existence of a chordless odd cycle of length greater than 5 , a contradiction.

Case 2. So suppose $Q$ leaves $\mathcal{S}_{1}$ one of $x_{1}$ or $x_{10}$, say without loss of generality by symmetry, via $x_{1}$. Since $\left\{x_{1}, x_{8}\right\}$ is a non-co-bridge pair, $Q$ must next traverse a bibridge of type $B_{1,3}$ or one of type $B_{1,11}$ (but not both).

Case 2.1. Suppose $Q$ traverses a bibridge of type $B_{1,3}$. There are then only two possibilities for the next bibridge encountered by $Q$. If this bibridge is of type $B_{3,4}$, the induced paths $x_{1} x_{2} x_{11} x_{12} x_{4}$ and $x_{1} x_{10} x_{9} x_{11} x_{12} x_{4}$ (both of which avoid vertex $x_{3}$ ) together imply the existence of a chordless odd cycle of length greater than 5 , a contradiction.

On the other hand, if this bibridge is of type $B_{3,8}$, the induced paths $x_{1} x_{2} x_{11} x_{9} x_{8}$ and $x_{1} x_{10} x_{9} x_{8}$ (both of which avoid $x_{3}$ ) then guarantee the existence of a chordless odd cycle of length greater than 5 , a contradiction.

Case 2.2. So $Q$ must traverse a bibridge of type $B_{1,11}$. But then since $\left\{x_{11}, x_{j}\right\}$ is a non-co-bridge pair for $j=3,4,7,8, Q$ must pass through a bibridge of type $B_{11,12}$. But in this instance, the induced paths $x_{1} x_{2} x_{3} x_{4} x_{12}$ and $x_{1} x_{2} x_{3} x_{8} x_{7} x_{12}$ (both of which avoid $x_{11}$ ) guarantee a chordless odd cycle of length greater than 5 , a contradiction.

Lemma 6.6: Let $G$ be a 3-connected internally-4-connected graph of girth 5 such that all odd cycles of length greater than 5 have a chord. Then if $G$ contains a subgraph isomorphic to graph $J_{2}, G \cong P_{10}$.

Proof: Suppose $G$ does contain $J_{2}$ as a subgraph. We will assume the labeling shown in Figure 3.2.

Suppose $G \nsucceq P_{10}$.

Claim 1: The subgraph $J_{2}$ is induced.

This is immediate by the girth five hypothesis.

Claim 2: For all $1 \leq i<j \leq 8, N_{i}^{\prime} \cap N_{j}^{\prime}=\emptyset$.

For all pairs $\{i, j\} \notin\{\{1,5\},\{3,7\}\}$, the result follows by the girth five hypothesis. Suppose $N_{1}^{\prime} \cap N_{5}^{\prime} \neq \emptyset$ and $u \in N_{1}^{\prime} \cap N_{5}^{\prime}$. Let $C=x_{1} x_{2} x_{6} x_{7} x_{8} x_{1}$. Then $N^{\prime}(C)$ contains 
the induced path $x_{3} x_{4} x_{5} u$ of length 3 , thus contradicting Lemma 5.4. Thus $N_{1}^{\prime} \cap N_{5}^{\prime}=\emptyset$ and, by symmetry, $N_{3}^{\prime} \cap N_{7}^{\prime}=\emptyset$. The Claim follows.

Claim 3: There exists no $N_{i}^{\prime}-N_{j}^{\prime}$ edge, for all $i \neq j$.

If $\{i, j\}=\{1,3\}$, we get the configuration $J_{1}$ as an induced subgraph, contradicting Lemma 6.5. If $\{i, j\}=\{1,4\}$, then together with the path $x_{1} x_{2} x_{6} x_{5} x_{4}$ we get a 7 -cycle, contradicting Lemma 2.3. Similarly, if $\{i, j\}=\{1,5\}$, using path $x_{1} x_{2} x_{3} x_{4} x_{5}$ we get a 7 -cycle, and if $\{i, j\}=\{2,4\}$, using path $x_{2} x_{6} x_{7} x_{8} x_{4}$, we get a 7 -cycle in violation of Lemma 2.3. The Claim follows for all other pairs $\{i, j\}$ by the girth five hypothesis and symmetry.

Claim 4: All non-adjacent pairs of vertices are well-connected and hence, by Claim 3 and Lemma 2.1, are non-co-bridge-pairs.

This is easily checked.

Claim 5: For all $i, i=1, \ldots, 8$, no bridge in $\mathcal{B}_{i}$ has an attachment in three distinct $N_{j}^{\prime}$ 's, $j \neq i$.

Suppose to the contrary, that $B \in \mathcal{B}_{i}$ has attachments in $N_{j}, N_{k}$ and $N_{\ell}$, where $i, j, k$ and $\ell$ are distinct. Since there are no triangles in $J_{2}$, some two of $x_{j}, x_{k}$ and $x_{\ell}$ must be non-adjacent. But then these two vertices form a co-bridge-pair, contradicting Claim 4 and proving Claim 5.

Now consider the set $\left\{x_{2}, x_{8}\right\}$ as a vertex cut in $J_{2}$. By the 3-connectivity of $G$, there exists a path joining $x_{1}$ to one of the vertices in $\left\{x_{3}, x_{4}, x_{5}, x_{6}, x_{7}\right\}$ which does not pass through $x_{2}$ or $x_{8}$. Let $Q$ be such a path of minimum length.

Since $x_{1}$ and $x_{i}$, for $i=3,4,5,6,7$, are non-co-bridge pairs, $Q$ must pass through bridges in $\mathcal{B}_{2} \cup \mathcal{B}_{8}$. Since $\left\{x_{2}, x_{8}\right\}$ is a non-co-bridge pair, $P$ must pass through exactly one bibridge of type $B_{1,2}$ or exactly one bibridge of type $B_{1,8}$, but not both. Say without loss of generality by symmetry, $Q$ traverses a bibridge of type $B_{1,2}$. Then $Q$ must pass through a bibridge of type $B_{2, j}, j \neq 1$. But by Claim 4 , the only possible bridges of this type are of types $B_{2,3}$ and $B_{2,6}$. In the former case, induced paths $x_{1} x_{8} x_{4} x_{3}$ and $x_{1} x_{8} x_{7} x_{6} x_{5} x_{4} x_{3}$ both avoid vertex $x_{2}$ and hence guarantee the existence of a chordless odd cycle of length greater than 5 , while in the latter case, induced paths $x_{1} x_{8} x_{7} x_{6}$ and $x_{1} x_{8} x_{4} x_{5} x_{6}$ both avoid $x_{2}$ and therefore imply the existence of a chordless odd cycle of length greater than 5. Thus in each instance we arrive at a contradiction and the Lemma is proved.

Thus as explained at the beginning of this section, we have proved the following result.

Lemma 6.7: Suppose $G$ is 3-connected, internally 4-connected, has girth 5 and every odd cycle of length greater than 5 has a chord. Let $C$ be a 5 -cycle in $G$. Then if $N^{\prime}(C)$ contains an induced path of length $2, G \cong P_{10}$. 


\section{Final Remarks.}

Thus we have reduced the general conjecture to the case in which for every 5-cycle $C$ in $G, N^{\prime}(C)$ consists of a matching together with an independent set. We can say a bit more, however. Let $C=x_{1} x_{2} x_{3} x_{4} x_{5} x_{1}$ and suppose $M$ is a matching in $N^{\prime}(C)$. Suppose without loss of generality that a matching edge $y_{1} y_{2} \in G\left[N^{\prime}(C)\right]$ is such that $y_{1} \sim x_{1}$ and $y_{2} \sim x_{3}$. Then if there were a second edge of $M$ with attachments at $x_{3}$ and $x_{5}$, we would have a 7 -cycle, contradicting Lemma 2.3. Hence by the girth 5 hypothesis and symmetry we may assume that $M$ can be partitioned $M=M_{1} \cup M_{2}$ where all edges in $M_{1}$ attach to $C$ only at $x_{1}$ and $x_{3}$, while those in $M_{2}$ attach only at $x_{2}$ and $x_{4}$ or else $M_{2}=\emptyset$.

\section{References}

[A] B. Alspach, Cayley graphs, Handbook of Graph Theory, CRC Press, Boca Raton, FL, 2004, 505-514.

[AGZ] B. Alspach, L. Goddyn and C-Q. Zhang, Graphs with the circuit cover property, Trans. Amer. Math. Soc. 344 (1994) 131-154.

[AH1] K. Appel and W. Haken, Every planar map is four colorable. I. Discharging, Illinois J. Math. 21 (1977) 429-490.

[AH2] K. Appel and W. Haken, Every planar map is four colorable. II. Reducibility, Illinois J. Math. 21 (1977) 491-567.

[BS] N. Biggs and D. Smith, On trivalent graphs, Bull. London Math. Soc. 3 (1971) 155-158.

[Bö] T. Böhme, On spatial representations of graphs, Contemporary Methods in Graph Theory, R. Bodendieck Ed., Mannheim, Wien, Zürich (1990) 151-167.

[BCN] A. Brouwer, A. Cohen and A. Neumaier, Distance-regular Graphs, SpringerVerlag, Berlin, 1989.

[CMRS] A. Cavicchioli, M. Meschiari, B. Ruini and F. Spaggiari, A survey on snarks and new results: products, reducibility and a computer search, J. Graph Theory 28 (1998) 57-86.

[F] D. Fulkerson, Blocking and anti-blocking pairs of polyhedra, Math. Programming 1 (1971) 168-194.

[GHR] T. Gaudin, J-C. Herz and P. Rossi, Solution du problème No. 29, Rev. Franc. Rech. Operationelle 8 (1964) 214-218.

[H] G. Haggard, Edmonds characterization of disc embeddings, Proc. Eighth Southeastern Conference on Combinatorics, Graph Theory and Computing Congress. Numer. XIX, Utilitas Math., Winnipeg, Man., 1977, 291-302.

[HoSi] A. Hoffman and R. Singleton, On Moore graphs with diameters 2 and 3, IBMJ. Res. Develop. 4 (1960) 497-504. 
[HoSh] D. Holton and J. Sheehan, The Petersen Graph, Australian Mathematical Society Lecture Series 7, Cambridge Univ. Press, 1993.

[I] A. Ivanov, Geometry of sporadic groups. I., Petersen and tilde Geometries, Encyc. Math. Appl., 76, Cambridge Univ. Press, 1999.

[IS] A. Ivanov and S. Shpectorov, Geometry of sporadic groups. II., Representations and Amalgams, Encyc. Math. Appl., 91, Cambridge Univ. Press, 2002.

[J] F. Jaeger, A survey of the cycle double cover conjecture, Ann. Discrete Math. 27 (1985) 1-12.

[KS] K. Kilakos and F. Shepherd, Excluding minors in cubic graphs, Combin. Probab. Comput. 5 (1996) 57-78.

[Ko] M. Kochol, Cubic graphs without a Petersen minor have nowhere-zero 5-flows, Acta Math. Univ. Comenian. (N.S.) 68 (1999) 249-252.

[KM1] K. Kutnar and D. Marušič, Hamilton cycles and paths in vertex-transitive graphs - current directions, Discrete Math. 309 (2009) 5491-5500.

[KM2] K. Kutnar and D. Marušič, Recent trends and future directions in vertextransitive graphs, Ars Math. Contemp. 1 (2008) 112-125.

[LS] J. Lauri and R. Scapellato, Topics in graph automorphisms and reconstruction, Cambridge Univ. Press, 2003.

[LR] C. Little and R. Ringeisen, On the strong graph embedding conjecture, Proc. Ninth Southeastern Conf. on Combinatorics, Graph Theory and Computing, Congress. Numer., XXI, Utilitas Math. Winnipeg, Man., 1978, 479-487.

[L1] L. Lovász, Problem 11, Combinatorial Structures and their Applications (Proc. Calgary Internat. Conf. (Calgary, Alberta 1969), Gordon and Breach, New York, 1970, 243-246.

[L2] L. Lovász, Matching structure and the matching lattice, J. Combin. Theory Ser. B 43 (1987) 187-222.

[M1] W. Mader, $3 n-5$ edges do force a subdivision of $K_{5}$, Combinatorica 18 (1998) 569-595.

[M2] W. Mader, An extremal problem for subdivisions of $K_{5}^{-}$, J. Graph Theory 30 (1999) 261-276.

[Pu] W. Pulleyblank, Matchings and extensions, Handbook of combinatorics, Elsevier, Amsterdam, 1995, 179-232.

[Po] P. Potočnik, Edge-colourings of cubic graphs admitting a solvable vertextransitive group of automorphisms, J. Combin. Theory Ser B 91 (2004) 289300 .

[RST1] N. Robertson, P. Seymour and R. Thomas, A survey of linkless embeddings, Graph structure theory (Seattle, WA, 1991), Contemp. Math. 147, Amer. Math. Soc., Providence, RI, 1993, 125-136. 
[RST2] N. Robertson, P. Seymour and R. Thomas, Petersen family minors, J. Combin. Theory Ser. B 64 (1995) 155-184.

[RST3] N. Robertson, P. Seymour and R. Thomas, Sachs' linkless embedding conjecture, J. Combin. Theory Ser. B 64 (1995) 185-227.

[Sa] H. Sachs, On spatial representation of finite graphs, Finite and infinite sets (Eger, 1981), Colloq. Math. Soc. János Bolyai, 37, North-Holland, Amsterdam, 1984, 649-662.

[Sc] R. Scapellato, Vertex-transitive graphs and digraphs, Graph Symmetry (Montreal, PQ, 1996), NATO Adv. Sci. Inst. Ser. C Math. Phys. Sci., 497 Kluwer Acad. Publ., Dordrecht, 1997, 319-378.

[Se1] P. Seymour, On multi-colourings of cubic graphs and conjectures of Fulkerson and Tutte, Proc. London Math. Soc. 38 (1979) 423-460.

[Se2] P. Seymour, Sums of circuits, Graph theory and related topics, Academic Press, New York-London, 1979, 341-355.

[Sz] G. Szekeres, Polyhodral decompositions of cubic graphs, Bull. Austral. Math. Soc. 8 (1973) 367-387.

[Ta] P. Tait, Remarks on the colouring of maps, Proc. Royal Soc. Edinburgh, Ser. A 10 (1880) 729.

[Th] R. Thomas, Recent excluded minor theorems for graphs, Surveys in combinatorics, 1999 (Canterbury), London Math. Soc. Lecture Note Ser. 267, Cambridge Univ. Press, 1999, 201-222.

[TT] R. Thomas and J. Thomson, Excluding minors in nonplanar graphs of girth at least five, Combin. Probab. Comput. 9 (2000) 573-585.

[Tu1] W. Tutte, On the algebraic theory of graph colorings, J. Combin. Theory 1 (1966) 15-50.

[Tu2] W. Tutte, A geometrical version of the four color problem (with discussion), Combinatorial Mathematics and its Applications (Proc. Conf. Univ. North Carolina, Chapel Hill, N.C., 1967), Univ. North Carolina Press, 1969, 553-560.

[Tu3] W. Tutte, A family of cubical graphs, Proc. Cambridge Phil. Soc. 43 (1947) 459-474.

[Wa] J. Watkins, Snarks, Graph Theory and its Applications East and West, New York Acad. Sci., New York, 1989, 606-622.

[WW] J. Watkins and R. Wilson, A survey of snarks, Graph theory, combinatorics, and applications. Vol. 2 (Kalamazoo, 1988), Wiley, New York, 1991, 1129-1144.

[Zhan] C-Q. Zhang, Integer flows and cycle covers of graphs, Marcel Dekker, New York, 1997. 